\title{
Direct measurements of ocean surface velocity from space: Interpretation and validation
}

\author{
Bertrand Chapron $^{1}$, Fabrice Collard ${ }^{2}$ and Fabrice Ardhuin ${ }^{3^{*}}$ \\ ${ }^{1}$ Laboratoire d'Océanographie Spatiale, Ifremer, Centre de Brest, 29280 Plouzané, France \\ ${ }^{2}$ BOOST-Technologies, 29280 Plouzané, France \\ ${ }^{3}$ Centre Militaire d'Océanographie, Service Hydrographique et Océanographique de la Marine, 29609 Brest, \\ France. \\ *: Corresponding author : ardhuin@shom.fr
}

\begin{abstract}
The median Doppler shift of radar echoes is analyzed in measurements by ENVISAT's Advanced Synthetic Aperture Radar (ASAR) over the ocean. This Doppler centroid differs from a predicted signal based on the predicted motion of the satellite and Earth. This anomaly, converted to a surface Doppler velocity U D , appears to be of geophysical origin. Two wide-swath images over the Gulf Stream around Cape Hatteras suggest that $U$ D contains high-resolution information on surface currents, while on a global scale, $U$ D is found to vary with the wind speed in the range direction. A simple quantitative forward model is proposed, based on a practical two-scale decomposition of the surface geometry and kinematics. The model represents the effect of the wind through the wave spectrum, and gives $U D \approx \mathrm{y} U 10\|+\mathrm{U}\| \|$, with $U 10 \|$ and $U \mathrm{c} \|$ as the $10 \mathrm{~m}$ wind speed and quasi-Eulerian current in the line of sight of the radar projected on the sea surface, respectively, and $\mathrm{y}$ as a coefficient function of the wind speed, wave development, and radar geometry. It is found that for an incidence angle of $23^{\circ}, y \approx 0.3$ for moderate winds and fully developed seas. This model is validated with a global data set of ASAR Wave Mode observations, with colocated model winds, acquired over the global ocean during the years 2003 and 2004. The Doppler signal therefore provides the signed parameter $U D$ that can be used to reduce the wind direction ambiguity in the inversion of high-resolution wind fields from SAR imagery. A qualitative validation of current effects is shown for the English Channel where tidal currents dominate. Thus it should be possible to combine this previously ignored geophysical Doppler signal with traditional information on sea surface roughness, in order to provide very high resolution wind and current fields.
\end{abstract}

Keywords: synthetic aperture radar; current Doppler; ENVISAT 


\section{Introduction}

Over the last 40 years numerous spaceborne optical and radar observations have revealed surface signatures related to the dynamics of the upper ocean at mesoscale and submesoscale [e.g. Fu and Holt, 1983; Johannessen et al., 1991, 1996; Munk et al. 2000]. Especially over the Gulf Stream region, both optical [Strong and Derycke, 1973] and high resolution radar observations [Ainsworth et al. 1995; Marmorino et al., 1997; Ufermann and Romeiser, 1999] exhibit complex and very well delineated mesoscale surface features related to the upper ocean circulation and dynamics. Contrasting reflectances and radar backscatter variations are known to follow surface roughness changes under varying surface winds and currents. In particular, current shear and convergence zones can focus wave energy resulting in intense wave steepening and enhanced radar backscatter.

Because roughness elements move over the ocean, radar measurements reveal Doppler shifts $f_{D}$ in the received echo frequencies [e.g. Keller et al., 1986]. Consequently, the Doppler signal should provide information on the surface kinematics, in complement to the radar backscatter which is related to the surface geometry. The frequency (or "Doppler") spectrum of returned signals has been extensively used to map surface currents with high-frequency (HF) radars [e.g. Barrick, 1972; Essen and Gurgel, 2000], for which the backscatter appears to be well predicted by Bragg scattering theory, at least for moderate sea states. In the microwave domain, Doppler spectra have been obtained from fixed platforms, providing evidence for the dominant role played by Bragg scattering [Plant and Keller, 1990; Thompson et al., 1991]. The Doppler spectra have also been used 
to measure the orbital velocity in surface gravity waves and study radar cross-section modulations [e.g. Feindt et al., 1986].

The first measurements of ocean surface kinematics from a moving platform used phase differences measured between two radar receiving antennas spaced along the platform track. These phase differences yields a surface velocity $U_{D}$ of the ocean surface, defined as the line-of-sight velocity projected on the mean sea surface [e.g. Chapman 1994]. Successful experiments with airborne radars [Shuchman, 1979; Goldstein and Zebker, 1987; Marom et al., 1990] as well as from the space Shuttle [e.g. Romeiser et al. 2003a] demonstrated that ocean currents and wave orbital velocities could be measured with two radar antennas. Because the surface current is a key variable of ocean dynamics, its measurement is highly desirable as a source of data for assimilation in ocean circulation models. New satellite missions that use this along-track interferometry (ATI) concept should be launched shortly [e.g. Romeiser et al. 2003b], providing data that could partially fill the gap left by some satellite altimeters, soon to be retired, in order to constrain numerical forecasts of the ocean circulation.

The surface velocity $U_{D}$ corresponds more precisely to a mean motion of scattering elements at the sea surface, averaged over the resolution of the radar image, with element velocities weighted by their normalized radar cross section (NRCS). This measurement clearly depends upon the instrument characteristics (wavelength, incidence angle, polarization state, antenna footprint). Besides, and similarly to the radar backscatter, several factors contribute to the measured Doppler velocity, including the desired bulk surface current velocity but also less known effects related to wave-current interactions at different scales. In particular Thompson et al. [1991] showed a large contribution to $U_{D}$ of the 
correlation between wave orbital velocities and NRCS for X-band radar at small incidence angle, for which the NRCS is highly sensitive to the incidence angle. We shall call this effect the tilt bias. ATI experiments uncovered some of the difficulties encountered in the interpretation of $U_{D}$. In cases with strong geostrophic or tidal currents $U_{D}$ appeared well correlated with surface currents [Graber et al. 1996; Romeiser et al. 2003], but other experiments showed that $U_{D}$ may be significantly different from the surface drift current [e.g. Shemer et al., 1993]. Clearly, the general geophysical interpretation of $U_{D}$ is not well established [Moller 1998].

In the case of HF radars, it has often been mentioned that the measured 'current' corresponds to a depth of some fraction of the surface wave wavelength that satisfies the Bragg resonant condition [Stewart and Joy, 1974], due to the effect of current shears on wave propagation velocities [White, 1999]. Yet the 'current' also contains some contribution of the wave-induced Stokes drift [Weber and Barrick, 1977; Broche et al., 1983]. Besides, it was also realized recently [Ardhuin et al. 2004; Rascle et al., paper in preparation for this journal] that the surface quasi-Eulerian current, defined as the drift current minus the wave-induced Stokes drift, should be rather uniform over the vertical due to strong wave-induced mixing [e.g. McWilliams et al. , 1997; Mellor and Blumberg, 2004]. This effect was observed by Santala and Terray [1992; see also Terray et al. 2000], and it suggests that most of the surface shear if often due to the Stokes drift. These effects could explain the observed "surface shear" seen in HF radar data by Fernandez et al. [1998]. In the microwave domain, other effects such as the tilt bias can contribute to $U_{D}$, but the radar is most sensitive to velocities much closer to the surface, say within $1 \mathrm{~cm}$ [Graber et 
al. 1996]. The detailed relationship of $U_{D}$ with properly defined Eulerian or Lagrangian currents [e.g. Peirson and Banner 2003] is yet to be fully explored.

Yet, one may not need to wait for new satellite missions to obtain routine measurements of $U_{D}$ from space. Here we demonstrate that a similar $U_{D}$ can be obtained from a detailed analysis of spaceborne synthetic aperture radar (SAR) single-antenna measurements. The technique used here relies on a careful analysis of the residual Doppler shifts in the frequency of the radar echoes. In satellite measurements, the centroid $f_{\mathrm{Dc}}$ of the measured Doppler spectrum is dominated by the platform along-track velocity relative to the rotating Earth. On ENVISAT, thanks to the very stable satellite orbit and attitude (rotations around the platform center of mass), it is possible to estimate the precise satellite motion and antenna pointing contributions $f_{\mathrm{Dcm}}$ to $f_{\mathrm{Dc}}$. Subtracting the modelled Doppler shift from the measured $f_{\mathrm{Dc}}$, yields a centroid anomaly $f_{\mathrm{Dca}}=f_{\mathrm{Dc}}-f_{\mathrm{Dcm}}$ that should be related to ocean surface motions only. For a simple target of fixed shape moving along the surface with a velocity $U_{D}$, defined positive when the target moves away from the radar, we should have

$$
U_{D}=-f_{\mathrm{ca}} / k_{e} \sin \theta_{I}
$$

with $k_{e}$ the electromagnetic wave number and $\theta_{I}$ the angle of incidence of the radar beam relative to the normal to the surface. Please note that this sign convention is identical to that chosen by Romeiser and Thompson [2000]. In principle this measurement of $U_{D}$ is a simple cross-section weighted average of the higher-resolution ATI-SAR measurements. Because the "Bragg" peaks familiar in HF radar signals are not distiguinshable due to other sources of motion, the centroid estimator (see Appendix A) picks the Doppler shift corresponding to the single and broad peak of the Doppler spectrum. Discussion on the 
representativeness of the obtained velocity [e.g. Graber et al., 1996] should also apply to our measurements (see also Appendix B).

A first demonstration of the technique was performed by van der Kooij et al. [2001] over the Gulf Stream, and the variations of $U_{D}$ within a SAR image acquired by ERS-1 was interpreted as a geostrophic current and validated with a ship-mounted ADCP. However, Chapron et al. [2004] revealed that the values of $f_{\text {Dca }}$ on a global scale are well correlated with winds at the surface of the ocean.

The goal of the present paper is to give a general geophysical interpretation of the surface velocity $U_{D}$ and propose a quantitative model relating $U_{D}$ to geophysical parameters. In section 2 we discuss results from a prototype grid of rather dense Doppler centroid estimates on Wide Swath (about $400 \mathrm{~km}$ ) SAR images provided by the European Space Agency to the Wide Swath Complex working group. In the future, the gridded values of $f_{\mathrm{Dca}}$ or $U_{D}$ could be provided as a metadata in every complex or detected product.

A quantitative interpretation of $U_{D}$ in terms of surface winds and currents is proposed in section 3, with a validation of the relationship between surface winds and Doppler velocities, extending the initial work of Chapron et al. [2004]. We further attempt a first validation of surface current signatures in section 4 , and give our conclusions in section 5 .

\section{Doppler and roughness signatures of the Gulf Stream}

The capabilities of the ENVISAT's Advanced SAR (ASAR) instrument are largely improved from the ERS-1/2 instruments, offering unique opportunities for Earth remote sensing. The first two examples of Wide Swath Complex images processed from ocean scenes are shown in figures 1 and 3 . Details of the processing performed to arrive at $f_{\text {Dca }}$ are given in Appendix $\mathrm{A}$, and the velocity $U_{D}$ follows from equation (1). In order to 
compensate for unknown biases due to the antenna mispointing angle (Appendix A), $f_{\text {Dca }}$ was adjusted to have a mean value of zero on land. Biases within swaths [e.g. Cafforio et al., 2004] where further adjusted by enforcing a continuous variation of $f_{\mathrm{Dca}}$ from one swath to the next using a third order polynomial fit to the observed $f_{\text {Dca }}$.

The resolution of the surface Doppler velocity $U_{D}$ in the flight direction is typically coarser than the real aperture antenna, because $f_{D c}$ must be estimated from the Doppler spectrum. Therefore the $U_{D}$ map is much coarser than the NRCS image, for which the Doppler information is already used in the aperture synthesis processing to provide the high azimuthal resolution (Appendix A). Therefore the present Doppler centroid method cannot achieve the resolution of the multiple-antenna along track interferometry systems. In practice, the resolution is further reduced by the use of multiple swaths for wide swath images.

Figure 1 illustrates the foreseen mapping capabilities with $U_{D}$ fields obtained from wide swath images acquired in vertical polarization (VV), at incidence angles $17^{\circ}<\theta_{I}<54^{\circ}$. The image was acquired on February 6 2003, at 15:02 UTC, along a descending track so that $U_{D}<0$ corresponds, with our convention, to surface velocities to the east-south-east (toward the satellite). $U_{D}$ was processed to a resolution of $25 \mathrm{~km}$ in azimuth and a varying resolution in range, coarser close to the satellite track. The NRCS $\sigma_{0}$ has a resolution of $100 \mathrm{~m}$. A broad and meandering area of easterly velocities (in red) lies immediately to the South of a sharp $\sigma_{0}$ gradient that is typical of oceanic thermal fronts [Johannessen et al. 1991; Marmorino et al. 1997]. This coincidence of $\sigma_{0}$ and $U_{D}$ features suggests that the area $U_{D}<0$ corresponds the the core of the Gulf Stream as it flows into the Atlantic after detaching from the coast at Cape Hatteras, and before it starts meandering 
and spinning off eddies. At the time of image acquisition, wind was from the North-East and increases with distance offshore, as revealed by buoy measurements of the National Data Buoy Center (NDBC) and the QuikScat instrument on board NASA's OceanWinds satellite (not shown). This wind pattern explains the weak $\sigma_{0}$ (dark areas) to the North of the image. $\sigma_{0}$ is also generally larger over the Gulf Stream (brighter area in figure 1), probably due to the higher relative wind speed that generates waves against the current, and the adjustment of the atmospheric boundary layer over the warm water.

Unfortunately, no sea surface temperature image is available to complement this picture because of a large cloud cover at that time. Yet, the position of the Gulf Stream is confirmed by the analysis of sea surface height ( $\mathrm{SSH})$, derived from satellite altimeter data over a one week window around the time of the SAR overpass (figure 2). The main $\mathrm{SSH}$ gradient and its meanders (figure 2) match the pattern of $U_{D}$ (figure 1). There is a possible correspondence between the cyclonic eddy seen in the SSH south of the Gulf Stream and the variation in $U_{D}$ along the $72^{\circ} \mathrm{W}$ meridian. However, smaller structures revealed by mushroom-type features in the NRCS do not have visible signatures in $U_{D}$ (e.g. in the area $37.5-38^{\circ} \mathrm{N}$ and $73-72^{\circ} \mathrm{W}$ ) probably due to the coarse resolution of $U_{D}$ and likely weaker surface velocities.

Figure 3 shows a processing of a second image with a finer resolution in azimuth (8 $\mathrm{km})$. The image was acquired on March 19, 2004 at 15:12 UTC. Winds were from the North-West in the northern part of the image (as confirmed by wind data from Cape Lookout station), and from the West in the southern part of the image, as found from wind data measured at Frying Pan Shoals (off Cape Fear). Areas of negative $U_{D}$ appear to meander along the capes (Cape Fear and Cape Lookout), south of Cape Hatteras. If 
$U_{D}$ is interpreted as the range component of the surface current then, upstream of Cape Hatteras, the Gulf Stream is not a single jet with a broad core uniform in the downstream direction. Instead, the current periodically meanders with Easterly flow reversals $\left(U_{D}>0\right)$ occuring at $\left(32.75^{\circ} \mathrm{N}, 77.5^{\circ} \mathrm{W}\right),\left(33.75^{\circ} \mathrm{N}, 76.5^{\circ} \mathrm{W}\right)$ and $\left(34.25^{\circ} \mathrm{N}, 75.5^{\circ} \mathrm{W}\right)$, and a region of strong Westerly flow on the shelf, to the West of $78^{\circ} \mathrm{W}$. The offshore jet is captured by the near real-time HYCOM 1/12 degree Atlantic Ocean prediction system (figure 4a) run by the Naval Research Laboratory and the Rosentiel School for Marine and Atmospheric Science (http://hycom.rsmas.miami.edu; Chassignet et al., manuscript submitted to Journal of Marine Systems) in the framework of the Global Ocean Data Assimilation Experiment (GODAE).

This offshore westerly jet is clearly related to the main temperature front on the continental shelf seen in MODIS sea surface temperature (SST) data (figure 4b), while the inshore Westerly velocities may be related to the more diffuse inshore SST gradient. Further, the Easterly velocities at $\left(32.75^{\circ} \mathrm{N}, 77.5^{\circ} \mathrm{W}\right),\left(33.75^{\circ} \mathrm{N}, 76.5^{\circ} \mathrm{W}\right)$ and $\left(34.25^{\circ} \mathrm{N}\right.$, $\left.75.5^{\circ} \mathrm{W}\right)$ appear to be related to warm water plumes stretched toward the West by cyclonic eddies on the shoreward side of the Gulf Stream (figure 4.b). Although these smaller scale motions are not reproduced by the HYCOM model, the SST features suggest that these Easterly surface velocities are probably real and that $U_{D}$ can be interpreted as the range component of the surface current.

Such images can be acquired by ENVISAT every 2 days on average for any part of the world ocean, and may be highly valuable for the Global Ocean Observing System (GOOS), allowing a high-resolution tracking of large current systems, even in the presence of cloud cover. As previously mentioned, the quantitative use of this information requires the 
capacity to understand and model both $U_{D}$ and $\sigma_{0}$. $U_{D}$ certainly contains information on surface currents, for which no other direct measurement is available from space, but $U_{D}$ is also modified by effects related to wind-wave-current interactions that shape the ocean surface geometry and velocity field at different scales. Measured values of $U_{D}$ should also depend upon how the ocean roughness is viewed by the radar, which includes effects of radar frequency, incidence angle, and polarization state.

\section{Doppler Model}

\subsection{Phenomenological analysis: global Doppler measurements}

To gain deeper understanding of the Doppler measurements and this mapping sensitivity, Chapron et al. [2004] used the opportunity provided by ENVISAT's ASAR continuous operation in Wave Mode, acquiring small images every $100 \mathrm{~km}$ along the satellite track with $\theta_{I}=23^{\circ}$. As an example we show data that cover one full orbit cycle of ENVISAT (i.e. 35 days) from January 1 to February 4, 2004 (this example was chosen because the southern hemisphere summer yields a minimum in sea-ice cover, and thus a wider extent of our data). The biases due to unknown antenna mispointings (Appendix A) were reduced by shifting the anomalies of each track (half-orbit), so that a sigmoid-shaped function fitted to the joint distribution of $U_{D}$ and $U_{10 \|}$ passed through the origin. Indeed the present analysis demands a stable estimate of the Doppler centroid $f_{\mathrm{Dc}}$ from one imagette to the next, which was not envisaged when the instrument was designed. This fitting procedure over each track has the merit of keeping the geophysical patterns in $f_{\mathrm{Dc}}$ while removing most of the jumps in $f_{\mathrm{Dc}}$ that were found to occur when the instrument the acquisition mode toggles between image and wave modes and the clock is reset. In order 
to remove large biases, due to strong inhomogeneities within one imagette (see Appendix A), imagettes were kept only when their normalized NRCS variances wasless than 1.4.

A typical global picture of the anomaly $f_{\text {Dca }}$ is shown in figure $5 \mathrm{a}$. Negative (westerly) values are generally observed at mid-latitudes and easterlies at tropical and polar latitudes, except for the northern Indian ocean and the Indonesian archipelago. That geophysical pattern appears closer to the broad patterns of the atmospheric circulation, rather than that of the ocean, with the much tighter Eastern boundary currents (Gulf Stream, Kuroshio, Agulhas current ...), except possibly in the equatorial Pacific where a rapid latitudinal variation is akin to the equatorial current system. However, converted to $U_{D}$, the Doppler anomaly is several times larger than expected oceanic currents. $U_{D}$ is tightly correlated to estimations of $U_{10 \|}$, the wind speed at $10 \mathrm{~m}$ height projected on the radar line of sight. This has been tested using co-located ECMWF weather analysis (figure $5 \mathrm{~b}$ ). For a given wind speed $U_{10 \|}$, the values of $U_{D}$ are distributed around a peak value close to $0.3 U_{10 \|}$, and a standard deviation less than $30 \%$ of this peak value, except at very low wind speeds. A saturation of $U_{D}$ at the highest wind speeds is also apparent. The same joint distribution of $U_{D}$ and $U_{10 \|}$ has been found continually for every orbit cycle from 2002 to 2004. Opposite signs of $U_{D}$ observed for descending and ascending tracks correspond to the same, nearly zonal, surface velocity component, viewed from almost opposite directions, except in polar regions.

The pattern of the satellite tracks (descending obliquely from the North-East to the South-West) is visible in figure 3b. This pattern, most pronounced at mid latitudes, is largely related to the variability of surface winds, but unknown biases in the Doppler processing may still be significant (see Appendix A). Thus, observations of $U_{D}$ for $\theta_{I}=23^{\circ}$ 
will be useful to improve the estimation of wind speeds and wind direction from SAR images, allowing to remove the 180 degree ambiguity in methods based on the NRCS value and its small scale texture [e.g. Portabella et al., 2002].

\subsection{A general Doppler model}

A detailed geophysical interpretation of $U_{D}$ now follows these global observations. Because $U_{D}$ is obtained with a resolution of a few kilometers, a model for that quantity necessarily involves an average over the phases of the surface gravity waves. We have seen that, to first order, an increase of the projected line-of-sight wind speed will increase both the measured radar cross section and the Doppler anomaly. The large $\left(30 \%\right.$ of $\left.U_{10}\right)$ value observed for $U_{D}$ suggests that, besides the surface drift velocity (about $3 \%$ of $U_{10}$ )

and the phase speed of Bragg waves (about $0.2 \mathrm{~m} \mathrm{~s}^{-1}$ ), longer and faster ocean waves are increasingly contributing to the overall radar cross section. Indeed, such a wind-correlated shift of the mean Doppler, weighted by the NRCS, was already anticipated by Winebrenner and Hasselmann [1988, see their equations 15 and 16e for the Doppler shift], and observed for similar incidence angles and shorter radar waves $\left(K_{u}\right.$ band) by Thompson et al. [1991]. These authors explained this observed shift as the result of correlations between the wave-induced NRCS modulations and the wave-induced surface velocities and corresponding Doppler shift. Their model, based on Bragg scattering and the modulation of Bragg waves by longer waves, gave a reasonable although underestimated value of $U_{D}$.

The complete description of the NRCS from statistical characteristics of the random sea surface is still an open question [e.g. Plant 2002, 2003; Elfouhaily and Guérin 2004]. Considering a pure resonant Bragg scattering mechanism, the NRCS should be determined entirely by the surface elevation spectrum at the resonant wavenumber. Under the 
geometrical optics approximation, scattering only depends on the probability distribution function of the surface slopes. More generally, under a Kirchhoff-like approximation, the radar cross section depends on the probability distribution function of the the joint elevation differences.

By analogy with the phenomenological approach introduced by Phillips [1985] to describe breaking wave statistics, the different individual scale contribution to the overall NRCS may be described by a distribution function $\Lambda(\vec{k}) . \Lambda(\vec{k}) d \vec{k}$ represents the relative contribution to the total radar cross section related to wave number in the range $\vec{k}$ to $\vec{k}+d \vec{k}$. A pure Bragg condition would lead to $\Lambda(\vec{k})=\delta\left(2 k_{e} \sin \theta_{I}\right) S(\vec{k})$, with $S$ the elevation spectrum. A geometrical optics approximation would give $\Lambda(\vec{k})=k^{2} S(\vec{k})$. Accordingly, the detected line-of-sight radar velocity follows as

$$
\bar{c}=\int c(\vec{k}) \Lambda(\vec{k}) \mathrm{d} \vec{k} / \int \Lambda(\vec{k}) \mathrm{d} \vec{k}
$$

In the geometrical optics approximation, the overall detected velocity is the mean surface slope velocities projected on the radar line-of-sight direction. At moderate to large incidence angles, specular reflections only account for a a very small fraction of the radar cross section, but the velocity of specular points may yield significant biases in the surface Doppler velocity [Winebrenner and Hasselmann, 1988].

A pure Bragg condition would yield for $\bar{c}$ a weighted mean of the phase velocity of the resonant scales. In real conditions, these short scales "Bragg scatterers" are running along longer surface waves, which are the only possible source for the large velocities found in the previous global analysis of ENVISAT imagettes (figure 5). Including these effects in a Bragg scattering model, both incidence angle and rotation of the incidence plane are deterministic functions related to the longer surface wave slopes. In such cases, the 
resonant wavenumber $\vec{k}$ can be different from the value on a flat surface, $\overrightarrow{k_{b}}=k_{e} \sin \theta_{I}$, and the distribution $\Lambda$ writes

$$
\Lambda(\vec{k})=\left[\frac{\left.\sigma_{0} \vec{k}\right)}{\sigma_{0}\left(\overrightarrow{k_{b}}\right)}\right] P(\vec{k})
$$

with $P$ the distribution function for the resonant conditions. This distribution is deduced from the probability distribution function of the longer wave slopes. The radar cross section $\sigma_{0}$ is evaluated at the resonant condition, and $k_{b}$ corresponds to the incidence angle on a flat ocean. Since longer resonant scales have larger phase velocity and elevation variances, the recorded Doppler shift corresponding to these Bragg contributions will increase with increasing slope of the long waves.

So far, the proposed model corresponds to rigid longer tilt modulations. In full generality, the phase velocity of the individual Bragg components shall be modified by the underlying longer wave motions. As seen by a stationary observer, the modifications follow perturbations of the dispersion relation for the short gravity-capillary waves by the orbital velocities and vertical accelerations of the larger scale components. In such a case, the distribution function of the resonant conditions shall include the joint probability distribution of slopes, velocities and accelerations, with randomly distributed phase velocities. The proposed model of the cross-section distribution over the surface scales, represented by their wavenumber $\vec{k}$, becomes

$$
\Lambda(\vec{k}, \overrightarrow{\dot{\eta}}, \ddot{\eta})=\left[\frac{\sigma_{0}(\vec{k})}{\sigma_{0}\left(\overrightarrow{k_{b}}\right)}\right] P(\vec{k}, \overrightarrow{\dot{\eta}}, \ddot{\eta})
$$


with $\overrightarrow{\dot{\eta}}$, and $\ddot{\eta}$ the local (horizontal and vertical) velocity and acceleration, respectively. And the mean line-of-sight velocity becomes

$$
\bar{c}=\frac{\iiint c(\vec{k}, \overrightarrow{\dot{\eta}}, \ddot{\eta})\left[\frac{\sigma_{0}(\vec{k})}{\sigma_{0}\left(\overrightarrow{k_{b}}\right)}\right] P(\vec{k}, \overrightarrow{\dot{\eta}}, \ddot{\eta}) \mathrm{d} \vec{k} \mathrm{~d} \overrightarrow{\dot{\eta}} \mathrm{d} \ddot{\eta}}{\iiint\left[\frac{\sigma_{0}(\vec{k})}{\sigma_{0}\left(\overrightarrow{k_{b}}\right)}\right] P(\vec{k}, \overrightarrow{\dot{\eta}}, \ddot{\eta}) \mathrm{d} \vec{k} \mathrm{~d} \overrightarrow{\dot{\eta}} \mathrm{d} \ddot{\eta}} .
$$

This development closely follows the derivations of the altimeter electromagnetic bias by Elfouhaily et al. [2001]. By analogy, the mean scatter velocity $\bar{c}$ could be called 'velocity bias'. $\bar{c}$ includes biases due to tilt and hydrodynamic modulations of the radar cross section. The main difficulty is to express the probability $P$ in a usable form. Romeiser and Thompson [2000] did not consider these latter elements and expressed a Doppler model using theoretical linear modulation transfer functions (MTF) between the surface elevation and the NRCS and Doppler shift.

\subsection{A simple practical Doppler model}

The general model may be simplified to allow a practical prediction of the measured Doppler shifts. Based on our global dataset, the principal mechanism that should be included, at least for $\theta_{I}=23^{\circ}$, is the Doppler biases resulting from correlations between the locally modulated radar cross section and the facet orbital motions. In particular, along a wave profile, facets tilted towards the satellite will generally appear brighter and move down (away from the satellite) for waves propagating away from the satellite (Figure 6), this tilt bias therefore contributes a surface velocity in the direction of the wave propagation. Further the short scale roughness is larger at the wave crests compared to the wave troughs, and slightly shifted toward the forward faces [e.g. Kudryavtsev et al. 2003b]. This hydrodynamic bias is thus also in the wave direction. Because Winebrenner 
and Hasselmann [1988] found that specular points may contribute large mean Doppler shifts, a model based on Bragg scattering alone may be too restrictive.

For numerical calculations of $\bar{c},(5)$ can be approximated using a two-scale decomposition of the ocean surface, allowing a simple parameterization of hydrodynamic modulation effects with semi-empirical expressions (Appendix 2). This analysis parallels that of Winebrenner and Hasselmann [1988], as repeated by Romeiser and Thompson [2000], with the only difference in the use of semi-empirical MTFs instead of theoretical MTFs in the latter study. It is found that the largest wave contributions arise from the high-frequency waves, proportional to the third moment of the surface elevation frequency spectrum. These short waves are closely related to the wind, and the surface Doppler velocity can be expressed as

$$
U_{D}=\bar{c}+U_{s \|} / 2+U_{c \|}
$$

where $U_{s \|} / 2$ and $U_{c \|}$ are half the surface Stokes drift (i.e. the mean wave-induced velocity at the surface for a fixed horizontal position) and quasi-Eulerian current (corresponding to the $\bar{u}^{L}-p$ variable of Andrews and McIntyre [1978]), in the direction of the line of sight projected on the mean sea surface.

Following standard developments, at second order in the wave slope (see Appendix B), the tilt contribution $T$ to $\bar{c}$ is proportional to moments of the elevation spectrum, namely,

$$
T=\frac{1}{\tan \theta_{I}} \frac{1}{\widehat{\sigma}_{0}} \frac{\partial \sigma_{0}}{\partial \theta} \int k_{\|} \omega S(\vec{k}) d \vec{k}
$$

with $\theta$ the incidence angle relative to the local tilted surface, and $S(\vec{k})$ the surface elevation spectrum [see also Romeiser and Thompson 2000]. Like the Stokes drift, $T$ is proportional to the third moment of the wave elevation frequency spectrum. 
For practical applications we use semi-empirically adjusted expressions [Kudryavtsev et al. 2003a, 2003b] for the tilt and hydrodynamic modulations. There are two main sources of uncertainty for the application of this model to the estimation of $U_{D}$. The first source of error is the exact shape of the high-frequency spectrum which may give significant differences in its third moment (i.e. the surface Stokes drift). The second source of error is the relatively poor knowledge of the MTFs.

In spite of these uncertainties, numerical evaluations of the proposed model account for most of the observed $U_{D}$ (figure $5 \mathrm{~b}$ ). With a surface Stokes drift of $1.6 \%$ of $U_{10}$, a root mean square difference of $0.3 \mathrm{~m} / \mathrm{s}$ is found between the model and the peak observed value of $U_{D}$ as a function of $U_{10 \|}$ (figure $5 \mathrm{~b}$, dashed line). However, realistic spectral shapes [Kudryavtsev et al., 1999] and in-situ measurements of surface bubble drift (J. Smith, personal communication, 2004) rather suggest a surface Stokes drift of $1.25 \%$ of $U_{10}$ for fully-developed waves. If this value of the Stokes drift is more realistic then the present model with the MTF of Kudryavtsev et al. [2003b] underestimates the measured velocity $U_{D}$. The effect of breaking wave fronts, possibly related to specular scattering, may account for that discrepancy, as suggested in the previous section.

In the present model, for $\theta_{I}=23^{\circ}$, the correlations of local vertical velocities and surface slopes yield the largest contributions to $U_{D}$. This tilt bias accounts for about $60-70 \%$ of $U_{D}$, depending on the choice of wave spectral shape and tilt MTF. This contribution may be interpreted as an 'amplified Stokes drift', with a gain factor controlled by the relative modulation of the radar cross section with incidence angle, i.e.

$$
G=\left(1 / \sigma_{0}\right)\left(\partial \sigma_{0} / \partial \theta\right)
$$


As found at $\theta_{I}=23^{\circ}, G$ can be as large as 15 for $U_{10}=7 \mathrm{~m} / \mathrm{s}$. For stronger winds, $G$ saturates as $\sigma_{0}$ gets less sensitive to the incidence angle. $G$ is also slightly asymmetric with larger values of $U_{D}$ for winds blowing away from or toward the radar. The hydrodynamic effects are found to be much smaller and, in the model, contribute less than $20 \%$ of $U_{D}$ for $\theta_{I}=23^{\circ}$.

Based on the measurements of Santala and Terray [1992], and personal communications of V. Kudryavtsev about measurements in the Black Sea, the downwind Eulerian mean surface current $U_{c \|}$ is of the order of $0.5 \%$ of $U_{10 \|}$. Reasonable spectra [Elfouhaily et al. 1997, Kudryavtsev et al. 1999] predict $U_{s}$ values between $0.008 U_{10}$ and $0.020 U_{10}$. Therefore the last contributing term is a mean horizontal drift velocity at the surface, $U_{c \|}+U_{s \|} / 2$, that should be close to $1.2 \%$ of $U_{10 \|}$ on average (assuming that the average removes geostrophic and tidal currents), less than half of the actual drift velocity of water particles in the open ocean [e.g. Huang, 1979; Csanady, 1984], and about 5\% of the measured $U_{D}$.

With this general understanding of the dominant contribution of short wind-waves to the Doppler velocity $U_{D}$, we can extend this result to different incidence angles. Using an empirical fit to the present dataset, the following relationship should be valid for radar frequencies in C-band and VV polarizations,

$$
\begin{aligned}
& U_{D, \text { empirical }}=0.022 G\left[1 .-0.52 \tanh \left(\frac{U_{10 \|}}{25}\right)\right] U_{10 \|} \quad \text { for } \quad U_{10 \|}>0 \\
& U_{D, \text { empirical }}=0.022 G\left[1 .-0.45 \tanh \left(\frac{-U_{10 \|}}{15}\right)\right] U_{10 \|} \quad \text { for } \quad U_{10 \|}<0,
\end{aligned}
$$

with the gain factor (8) assumed to be dominated by the tilt bias, and estimated heuristically from, e.g., the CMOD empirical model using the proper wind direction $\phi$ [Quilfen et al. 1998]. On the present global dataset, the r.m.s. difference is $0.84 \mathrm{~m} \mathrm{~s}^{-1}$ between 
equation (9) with the CMOD NRCS, using ECMWF winds, and the measured Doppler (keeping only those imagettes with normalized variances less than 1.4 and acquisition times within 1.5 hours of the ECMWF output). This empirical model predicts that the wave-related contributions to $U_{D}$ shall decrease with incidence angle according to the observed change of the gain factor. For $U_{10}=7 \mathrm{~m} \mathrm{~s}^{-1} G$ decreases from 14 at $\theta_{I}=23^{\circ}$ to 2 at $\theta_{I}=45^{\circ}$. For these larger incidence angles the hydrodynamic bias is likely to be larger than the tilt bias.

Following this approach, we can anticipate that for $\mathrm{HH}$ polarizations $G$ is larger, particularly so at higher incidence angles. Consequently, measured Doppler velocity will also be larger, and differences between $\mathrm{VV}$ and $\mathrm{HH}$ data can be foreseen to provide direct valuable information about the local Stokes drift component and wave-induced biases in $U_{D}$

It is rather surprising that the large influence of the wind speed has not been reported in previous ATI experiments. Yet, many authors have reported that, for technical reasons, they had to correct for the mean phase shift (i.e. the mean surface velocity). Thus they discussed only the relative values of $U_{D}$ [e.g. Ainsworth et al., 1995; Graber et al., 1996]. Further, the gain factor can be much smaller for other incidence angles and frequencies. In particular, it should be one order of magnitude smaller than here for the conditions observed by Plant and Keller [1990] in the North Sea, with a X-band radar and $40<\theta_{I}<65^{\circ}$, showing little, if any, mean Doppler shift.

\section{Coastal tidal current observations}

Now considering the contribution of surface currents to $U_{D}$, we only get a direct effect of the Eulerian current velocity $U_{c}$ via the last term of (5). Nevertheless, $U_{c}$ may also modify 
$U_{D}$ in several indirect ways. Firstly, $U_{10 \|}$ in (9) should be replaced by $U_{10 \|}-U_{c \|}$. Indeed, the apparent wind in a frame of reference moving with the current is the proper velocity for scaling the growth of wind waves, and the resulting roughness. Secondly, horizontal variations of $U_{c}$ modify the local wave kinematics and the higher frequency tail of the elevation spectrum. Thus, both the third order spectral moment, and the sensitivity gain factor $G$ shall be affected.

Therefore any Doppler velocity map such as figure 1 combines effects of the wind and current patterns in a rather complicated way. Besides, temperature gradients that give rise to currents, such as the Gulf Stream, also induce local changes in the wind field which makes the geophysical interpretation of $U_{D}$ even more delicate [e.g. Chelton et al. 2004].

In order to avoid some of these difficulties, we consider currents in the English Channel, where strong tidal motions maintain a uniform sea surface temperature in winter. In that area, a reasonably uniform wind field permits the investigation of the contribution of $U_{c}$ to $U_{D}$. Further, $U_{c}$ is generally well represented by numerical models of tidal currents. Here we use estimations of tidal currents based on the finite-element model TELEMAC2D [Galland et al., 1991], used for the production of tidal current atlases at the Service Hydrographique et Oceanographique de la Marine. Hourly model results for mean neap and spring tides were interpolated to the actual tidal amplitude and phase.

A total of six narrow swath Single Look Complex SAR images acquired by ENVISAT were processed. The processing was done by tiling the image as almost squared imagettes with a resolution of $2 \mathrm{~km}$. Each imagette was then processed to infer winds, waves and Doppler velocities. Wind and waves were validated with in-situ data [Collard et al., 2005; see also figure 2 in Ardhuin et al. 2005], and, again, the Doppler centroid anomaly for 
each imagette was defined by its Doppler centroid minus the average Doppler centroid of imagettes on land. This definition was preferred to the one used for the global dataset in order to remove large scale biases.

A first image was acquired in VV polarization on March 92003 at 10:22 AM (UTC), with $31<\theta_{I}<37^{\circ}$ (figure 7a). $U_{D}$ maps, obtained with a $2 \mathrm{~km}$ resolution, exhibit a qualitative correlation with the tidal current in the line of sight, $U_{c \|}$ (figure $7 \mathrm{~b}$ ). In particular, strong Westerly flow is found North of Cherbourg and to the West of the Channel Islands (see figure 8 for locations).

Yet, local variations suggest that $U_{D}$ is not only due to tidal currents. As discussed in Appendix A, variations in the NRCS can also produce biases. Changes in the wave spectrum may also contribute to $U_{D}$. At the time of acquisition, a large swell $(4 \mathrm{~m}$ significant wave height $H_{s}$ ) was propagating from the West into the area of the image (figure 8). This swell should contribute to $U_{D}$ through the tilt and hydrodynamic biases (Appendix B). The relevant wave parameter is the swell-induced Stokes drift that may be estimated from directional buoy measurements south of Jersey (the largest island here, located at $49^{\circ} 12^{\prime} \mathrm{N}$ and $2^{\circ} \mathrm{W}$ ), where SAR-derived wave heights are within $10 \%$ of in-situ measurements [Collard et al., 2005]. This Stokes drift is $2 \mathrm{~cm} \mathrm{~s}^{-1}$ at the buoy where $H_{s}=2.3 \mathrm{~m}$, for waves in the range $0.05<f<0.27 \mathrm{~Hz}$ (the wind sea peak, excluded in this calculation, is at $0.28 \mathrm{~Hz}$ for that site).

Assuming a linear transformation of the entire spectrum to match the SAR-derived wave heights, waves twice as large West of Jersey yield a swell-induced Stokes drift four times as large in that area, i. e. $U_{s}=8 \mathrm{~cm} \mathrm{~s}^{-1}$. Please note that this linear transformation is likely to overestimate the Stokes drift amplification since the low-frequency waves are 
most affected by refraction and shoaling while shorter waves may adjust to the wind direction [e.g. van Vledder and Holthuijsen, 1993]. The change of $U_{D}$ downwave of Jersey may be related the observed change of the long waves field with very small waves observed to the East of the island, Using the C-MOD relation as in equation (9), we find a gain factor $G=6$ at $\theta_{I}=35^{\circ}$ and $U_{10}=7 \mathrm{~m} \mathrm{~s}^{-1}$. The strong swell is thus expected to contribute $G U_{s \|}$, of the order of (although likely less than) $48 \mathrm{~cm} \mathrm{~s}^{-1}$ to the observed velocity $U_{D}$ to the East of the islands, and near-zero in the immediate lee of the islands. This contribution may explain the discrepancy between the observed $1.2 \mathrm{~m} \mathrm{~s}^{-1}$ difference in $U_{D}$ between the area North of Cherbourg and East of Jersey, viewed with the same incidence angle, and the difference of $0.8 \mathrm{~m} \mathrm{~s}^{-1}$ expected from tidal currents variations.

Another image (figure 7c) was acquired in horizontal polarization ( $\mathrm{HH}$ ) in a similar tide-dominated environment. As expected from the model, $U_{D}$ appears to vary with the incidence angle, in the look direction. Although $U_{D}$ estimates are more noisy, a clear correlation is found between the local current and strong Doppler gradients, in particular around the islands and in the narrow entrance to the bay of Brest (figure 7d).

For these different cases, local gradients of the radar cross section are often related to strong currents. Without the Doppler information, the radar cross section variations would be wrongly attributed to gradients in the surface wind field only. This shows that Doppler measurements significantly advance the quantitative interpretation of current features manifested in SAR images, even at relatively coarse resolutions. More data will be needed to investigate the likely different effects of wind following or opposing the current on the local surface geometry (long and short scales), and the expected modulation of $U_{D}$ with the fetch. It is possible that the different properties of young waves found at short 
fetch will require a generalization of the semi-empirical MTFs used here. In particular, as a larger fraction of the ocean is covered by whitecaps and waves are generally steeper, the linear wave theory for the surface velocities used in Appendix B may require extensions to take into account the nonlinear kinematics of steep or breaking waves [e.g. LonguetHiggins 1987, Melville and Rapp 1988] and explicit modelling of specular point motion [Longuet-Higgins, 1957].

A first attempt was made at the estimation of surface current on a regular grid with 2 degree resolution, using the global data set of imagettes and equation (9). The result showed that the biases in the predicted Doppler largely dominated the result. Corrected mean velocities tended to be Easterly in places where western boundary currents such as the Gulf Stream and Kuroshio are expected, possibly due to the different sea-state and fetch conditions. Further, western boundary currents are so narrow that they disappear in such coarse spatial averages. Wide-swath images appear better suited for imaging these currents.

\section{Conclusion}

The present paper confirms initial demonstrations by Chapron et al. [2004] of the geophysical nature of Doppler centroid anomalies recorded by the Advanced Synthetic Aperture Radar (ASAR) instrument onboard ENVISAT. This information can be obtained from any SAR system using the Doppler centroid grid used for the SAR processing, or, alternatively, complex SAR images. However, the geophysical interpretation of the absolute Doppler centroid is only possible if the attitude of the platform is very well known. Otherwise, it seems that any system is able to provide at least relative values of the 
Doppler centroid, and thus gradients of $U_{D}$ within one image as demonstrated with both ERS-1 and RADARSAT data by van der Kooij et al. [2001].

Doppler velocities $U_{D}$ were obtained at resolutions of 2 or $8-25 \mathrm{~km}$, from narrow or wide swath images, respectively. Uncertainties in the measurements are not well known (Appendix A), and corrections for mean Doppler biases had to be performed, specific to the image mode used (wide swath, narrow swath, imagettes). Wide swath images over the Gulf Stream show, without ambiguity, a Doppler signature of the surface current.

A general theory for Doppler centroid measurements is proposed, and a simplified quantitative model is derived for the interpretation of the measured velocity in terms surface winds and currents. The contribution of the wind to $U_{D}$ is well predicted by the model, as shown by the validation using a global dataset of images co-located with ECMWF model winds. This validation demonstrates that wind induced Doppler velocities are signed, and dominated by the high frequency tail of the ocean surface elevation spectrum. This yields velocities $U_{D}$ of the order of $30 \%$ of the wind speed for incidence angles of $23^{\circ}$ in C-band, typically much larger than oceanic currents. It therefore appears difficult to estimate surface currents from a collection of imagettes, as shown in figure 5. Yet, the Doppler information can thus be used to improve the estimation of wind speeds and, more importantly, wind direction from SAR imagery. Over larger images, geostrophic currents that typically vary on smaller scales than the wind field can be imaged with a higher resolution than estimates derived from satellite altimetry. The accuracy of the measured velocity, such as previously reported by van der Kooij et al. [2001], is still to be further examined. 
Two narrow-swath images over coastal areas where tidal currents are well known are analyzed here. The effects of currents in the most simple use of the present model gives an overall good estimation of $U_{D}$. However, these two examples have important sea states with significant effects on the measured $U_{D}$. Therefore a precise estimation of oceanic currents will certainly require a better estimation of the wave-current interaction phenomena and knowledge of the local wave field. In principle the same quantity is measured with the Doppler centroid method and the Along-Track Interferometry, so that some of the processing could follow Romeiser and Thompson [2000]. Since local wave effects on the Doppler velocity should be a function of polarization and incidence angle (via the gain factor $G$ ), future SAR or ATI missions may use the expected polarization effects on both radar cross section and Doppler measurements to estimate the wind-related Doppler contributions and thus obtain the proper correction for current measurements. The use of star-trackers for attitude measurements on future SAR satellite platforms may greatly reduce the uncertainties in attitude that appear to cause most of the error on absolute values of $U_{D}$.

Beyond obvious applications to measurements of surface winds and currents, the Doppler velocity can also supplement the high-resolution wave information traditionally extracted from SAR images. This combination should help the investigation of dangerous "freak" waves that are known to be more frequent in areas with particular configurations of surface currents [e.g. Lavrenov 2003]. ENVISAT's ASAR Doppler data, when its quantitative interpretation is improved, has a unique capability for a multiscale monitoring (global or local) of the ocean surface velocity field. Besides, this new measurement 
can be combined together with the detection of even finer frontal structures using radar or other remote sensing techniques.

\section{Acknowledgments.}

We would like to acknowlege the technical support provided by the European Space Agency (ESA), in particular, B. Rosich, B. Duesmann and M. Zink. Imagettes and SLC products are property of ESA and were provided by Y.-L. Desnos, B. Rosich, and R. Cordey. Processing for the Wide Swath images follows developments by A. Garnieri (Politecnico di Milano). Model winds were provided by ECMWF as part of ESA's cal/val activities, and model currents were obtained from Internet Live Access servers thanks to the Global Ocean Data Assimilation Experiment (GODAE), while in-situ data was obtained from NDBC's internet on-line archives. We thank V. Kudryavtsev and N. Reul for discussing the results.

\section{Appendix A: Estimation of the Doppler Centroid frequency anomaly}

The technique used here relies on a careful analysis of the residual Doppler shifts in the frequency of the radar echoes. An accurate estimation of this Doppler Centroid frequency anomaly requires both the estimation of the Doppler centroid frequency $f_{\mathrm{Dc}}$ and the

prediction $f_{\mathrm{Dcm}}$ of the Doppler centroid frequency for a non-moving target in the earth reference frame.

\section{A1. Doppler centroid estimation}

$f_{\mathrm{Dc}}$ is used to perform the aperture synthesis and is thus commonly estimated in standard SAR processing. Discussions on the accuracy of $f_{\mathrm{Dc}}$ estimation methods have been the subject of many papers, recently reviewed by $Y u$ and $Z h u$ [1997]. $f_{\mathrm{Dc}}$ estimation errors 
are typically proportional to the inverse of the range/azimuth image block size over which the estimation is performed. E.g. for satellite SARs and an image block of 32(range) $\mathrm{x}$ 2048(azimuth) pixels (about 500m by $8 \mathrm{~km}$ for ENVISAT), the error is typically of the order of 4 to $10 \mathrm{~Hz}$ depending on the backscatter homogeneity. For the imagettes products delivered by ESA (figure 5), the Doppler centroid is included in the header. This was not the case for wide swath images, for which the Doppler centroid was provided separately to the authors.

But $f_{\mathrm{Dc}}$ can also be estimated on high resolution complex SAR images (i.e; after aperture synthesis). This was performed on the narrow swath images: the Doppler spectrum is obtained by Fourier transform of the complex image in the azimuth direction over blocks equally spaced in azimuth, and the resulting spectra are averaged over several range pixels to obtain a stable spectral estimate. If the Doppler estimate is accurate enough, small block sizes are possible, down to an azimuth size of the order of the real antenna footprint. The centroid of the Doppler spectrum is then obtained using the centroid estimator of Madsen [1989], identical to the one used by ESA for the wide swath and imagette products. The accuracy of the Doppler centroid from the complex image is further limited by the initial processing of the SAR image [see also Cafforio et al., 2004]. Due to filtering in that processing, a "scalloping" pattern, regular in the azimuthal direction, is evident in figure 7. Ideally the $f_{\mathrm{Dc}}$ grid should therefore be supplied together with the image.

\section{A2. NRCS gradients and $f_{\mathrm{Dc}}$ errors}

Because of the strong Doppler variations across the antenna lobe, a gradient in NRCS will bias $f_{\mathrm{Dc}}$ to the Doppler of the brighter part of the illuminated ground [e.g. Cumming, 2004]. This effect is most pronounced in areas of weak NRCS (to the South of the image in 
figure 3, or sharp NRCS gradients (along the coast to the South of Cape Fear in the same image). The values of $f_{\mathrm{Dc}}$ estimated here could be further improved in areas of strong NRCS gradients, by compensating for the Doppler frequency bias introduced by a rapid azimuthal NRCS variation. This correction can be estimated by applying (to the high resolution azimuthal backscatter variation obtained after SAR processing) the theoretical bias of the $f_{D c}$ estimator used. Simulations for ENVISAT suggest that a relative increase in NRCS by a factor 4 could cause a bias of $100 \mathrm{~Hz}$ in $f_{\mathrm{Dc}}$, over the area of the block used in the average. Such an error is of the order of the measured signal. Yet, the NRCS is much more uniform over most of the images in figure 1 and 3 with typical variations of the order of $10 \%$ over a block, resulting in expected biases of the order of a few Hertz.

\section{A3. Satellite attitude and $f_{\mathrm{Dc}}$ errors}

However, in satellite measurements, $f_{\mathrm{Dc}}$ is dominated by the platform velocity relative to the rotating Earth projected on the antenna pointing direction. Whereas earth motions relative to the satellite platform are rather easily predictable over the sea (a rigid body rotation of a perfect ellipsoid is accurate enough), the antenna pointing angle are generally not known with enough accuracy and not stable enough to ensure Doppler prediction within less then $10 \mathrm{~Hz}$. As an illustration of the sensitivity relative to platform attitude, a change of 10 millidegree in the yaw angle will cause a $50 \mathrm{~Hz}$ shift in the Doppler prediction. On ENVISAT, thanks to the very stable satellite orbit and predictable attitude (yaw pitch and roll), it is possible to estimate the precise contribution of satellite motion and antenna pointing $f_{D c m}$ to the Doppler centroid. Except for an unknown bias, the predicted attitude is expected to be accurate within a few millidegrees (B. Duesman, personal communication, 2005). 
Subtracting the modelled Doppler shift from the measured Doppler centroid, yields a centroid anomaly $f_{\mathrm{Dca}}=f_{\mathrm{Dc}}-f_{\mathrm{Dcm}}$ that should be related to ocean surface motions only. Although the predicted satellite attitude and velocity are hardly accurate enough to reveal this geophysical signal on a global scale, these orbital effects vary smoothly so that within one image (figures 1, 3 and 7 ), the relative surface velocity is certainly more accurate. The use of restituted (measured) orbital parameters could, in the future, significantly improve the accuracy the estimates of $U_{D}$ on a global scale.

\section{Appendix B: Derivation of the Doppler velocity using radar cross section modulations}

Without specifying the details of the backscatter from the sea surface, we use a twoscale approach in which the radar cross section can be defined over a collection of rough facets. The position, velocity and slope of these facets are described with a superposition of surface gravity waves with random phases. The salient feature of the two-scale approach is that the local radar cross section is modulated according to the local instrument geometry and environmental conditions, and $U_{D}$ is obtained by an average over the random wave phases. The Doppler centroid frequency anomaly $f_{\text {Dca }}$ divided by the electromagnetic wavenumber $k_{e}$ represents a mean velocity of surface rough facets towards or away from the SAR antenna. Assuming that $f_{\text {Dca }}$ is a simple geometrical mean weighted by the normalized radar cross section of each surface element, we have the facet velocity along the line of sight,

$$
\frac{2 \pi f_{\text {Dca }}}{k_{e}}=\frac{\overline{\left(u \sin \theta_{I}-w \cos \theta_{I}\right) \sigma_{0}\left(\theta_{I}+\Delta \theta\right)}}{\overline{\sigma_{0}\left(\theta_{I}+\Delta \theta\right)}}
$$

where $u$ and $w$ are the horizontal (in the range direction) and vertical velocities of the surface elements, the overbar denotes an average over horizontal space, and $\Delta \theta$ is the 
local modification of the incidence angle $\theta_{I}$, determined from the scalar product of the local normal to the surface and the unit vector along the incident direction. At second order in the wave slope $\varepsilon$, with $\eta$ the surface elevation,

$$
\Delta \theta=-\frac{\partial \eta}{\partial x}+\frac{1}{2 \tan \theta_{I}}\left\{\left[1+\sin \theta_{I}\right]\left(\frac{\partial \eta}{\partial x}\right)^{2}+\left(\frac{\partial \eta}{\partial y}\right)^{2}\right\}+O\left(\varepsilon^{3}\right)
$$

First ignoring hydrodynamic modulations of the roughness we may write

$$
\sigma_{0}\left(\theta_{I}+\Delta \theta\right)=\overline{\sigma_{0}}+\Delta \theta \frac{\partial \sigma_{0}}{\partial \theta}+\frac{\Delta \theta^{2}}{2} \frac{\partial^{2} \sigma_{0}}{\partial \theta^{2}}+O\left(\varepsilon^{3}\right)
$$

Using linear wave theory, for a monochromatic wave train of amplitude $a$, wavenumber $\left(k_{x}, k_{y}\right)$, intrinsic radian frequency $\omega$ and phase $\phi=\left[k_{x} x+k_{y} y-\left(k_{x} U_{c x}+k_{y} U_{c y}+\omega\right) t\right]$ we have, at the surface, with a quasi-Eulerian current $U_{c}$ and Stokes drift $U_{s}$,

$$
\begin{aligned}
\eta & =a \cos \phi+A_{2} \sin (2 \phi)+O\left(\varepsilon^{3}\right) \\
w & =a \omega+\sin \phi+W_{2} \sin (2 \phi)+O\left(\varepsilon^{3}\right) \\
u & =U_{c x}+\frac{1}{2} U_{s x}+a \omega \cos \phi+U_{2} \cos (2 \phi)+O\left(\varepsilon^{3}\right) \\
\omega^{2} & =g k \tanh (k H) \\
U_{s x} & =a^{2} k_{x} \omega \frac{\cosh (2 k H)}{2 \sin ^{2}(k H)}+O\left(\varepsilon^{3}\right)
\end{aligned}
$$

where $H$ is the local water depth, and $U_{2}$ is the second order Stokes wave velocity. For $k H \gg 1$, one gets

$$
U_{s x} \equiv U_{s x}^{\infty}=k_{x} \omega a^{2}
$$

We can now rewrite (B1) as,

$$
\begin{aligned}
\frac{2 \pi f_{\text {Dca }}}{k_{e}} & =\frac{1}{\widehat{\sigma}_{0}}\left\{w \frac{\partial \eta}{\partial x} \cos \theta_{I} \frac{\partial \sigma_{0}}{\partial \theta}+\left[U_{c x}+\frac{1}{2} U_{s x}\right] \widehat{\sigma}_{0} \sin \theta_{I}\right\}+O\left(\varepsilon^{3}\right) \\
& =\frac{1}{\widehat{\sigma}_{0}}\left\{\frac{\sigma k a^{2}}{2} \frac{\partial \sigma_{0}}{\partial \theta} \cos \theta_{I} \frac{\partial \sigma_{0}}{\partial \theta}+\left[U_{c x}+\frac{1}{2} U_{s x}\right] \widehat{\sigma}_{0} \sin \theta_{I}\right\}+O\left(\varepsilon^{3}\right)
\end{aligned}
$$


with

$$
\widehat{\sigma}_{0}=\bar{\sigma}_{0}+\frac{1}{2} \overline{\left(\frac{\partial \eta}{\partial x}\right)^{2} \frac{\partial^{2} \sigma_{0}}{\partial \theta^{2}}}
$$

Calculating the correlations, one finds $\overline{w \partial \eta / \partial x}=U_{s x}^{\infty} / 2$. Then, generalizing the calculation to random waves, the wave-induced terms of different components add up because they are second order terms. One thus gets,

$$
\frac{2 \pi f_{\text {Dca }}}{k_{e}}=U_{s x}^{\infty} \cos \theta_{I} \frac{1}{\widehat{\sigma}_{0}} \frac{\partial \sigma_{0}}{\partial \theta}+\left[U_{c x}+\frac{1}{2} U_{s x}\right] \sin \theta_{I}+O\left(\varepsilon^{3}\right)
$$

and the horizontal Doppler velocity is, at second order,

$$
U_{D, \text { model }}=\frac{U_{s x}^{\infty}}{2 \tan \theta_{I}} \frac{1}{\widehat{\sigma}_{0}} \frac{\partial \sigma_{0}}{\partial \theta}+\left[U_{c x}+\frac{1}{2} U_{s x}\right]
$$

The derivation is equivalent, for the tilt effects, to the results of Romeiser and Thompson [2000, see their equations 10 and 14].

In order to represent the hydrodynamic modulation of the roughness over the phase of the long waves, we assume a known complex modulation transfer function $\alpha=\alpha_{r}-\mathrm{i} \alpha_{i}$, so that (B3) becomes

$$
\sigma_{0}\left(\theta_{I}+\Delta \theta\right)=\overline{\sigma_{0}}\left\{\left(1+\Delta \theta \frac{\partial \sigma_{0}}{\partial \theta}\right)+[1+|\alpha| k a \cos (\phi+\psi)]\right\}+O\left(\varepsilon^{2}\right)
$$

With $\psi$ the argument of $\alpha$. Detailed mechanisms of the modulation are rather complex [Kudryavtsev et al. 2003a,b] but the overall effect is a pronounced increase of the roughness at the wave crests, slightly on the forward face, essentially related to a stronger wind stress over the crests. Therefore, with our notations $\alpha_{r}$ and $\alpha_{i}$ are generally both positive. The correlation of the roughness in phase with the wave elevation (represented by $\alpha_{r}$ ) tends to increase the Doppler velocity $U_{D}$, while the correlation of the roughness with the forward wave slope (represented by $\alpha_{i}$ ) only increases the magnitude of $U_{D}$ for waves propagating 
towards the satellite,

$$
U_{D, \text { model }}=\frac{1}{2 \tan \theta_{I}}\left[-U_{s x}^{\infty} \frac{1}{\sigma_{0}} \frac{\partial \sigma_{0}}{\partial \theta_{I}}+\alpha_{i}\left|U_{s x}^{\infty}\right|\right]+\frac{\alpha_{r}}{2} U_{s x}^{\infty}+\left(U_{c x}+\frac{U_{s x}^{\infty}}{2}\right)
$$

If no detailed wave information is available, one may further parameterize $U_{s x}^{\infty}$ from the wind $x$-component at ten meters $U_{10 x}$

$$
U_{D, \text { model }}=\frac{1}{2 \tan \theta_{I}}\left[-\beta U_{10 x} \frac{1}{\sigma_{0}} \frac{\partial \sigma_{0}}{\partial \theta_{I}}+\alpha_{i} \beta\left|U_{10 x}\right|\right]+\frac{\alpha_{r}}{2} \beta U_{10 x}+\left(U_{c x}+\frac{\beta U_{10 x}}{2}\right) .(
$$

This expression is of the form

$$
U_{D, \text { model }}=G \beta U_{10 x}+\left(U_{c x}+\frac{\beta U_{10 x}}{2}\right)=\gamma U_{10 x}+U_{c x}
$$

in which $G$ is a gain factor that amplifies the third moment of the wave spectrum and biases the the Doppler velocity, and $\gamma$ is a general wind factor. Note that for vertically sheared currents, the value of $U_{c x}$ that appears in the facet velocity should be weighted by the profile of the Stokes drift, which is the appropriate Doppler shift for waves on a sheared current [White, 1999].

\section{References}

Ainsworth, T. L., S. R. Chubb, R. A. Fusina, R. M. Goldstein, R. W. Jansen, J.-S. Lee, and G. R. Valenzuela (1994), INSAR imagery of surface currents, wave fields and fronts, IEEE Trans. on Geosci. and Remote Sensing, 33(5), 855-865.

Andrews, D. G., and M. E. McIntyre (1978), An exact theory of nonlinear waves on a Lagrangian-mean flow, J. Fluid Mech., 89, 609-646.

Ardhuin, F., F.-R. Martin-Lauzer, B. Chapron, P. Craneguy, F. Girard-Ardhuin, and T. Elfouhaily (2004), Dérive à la surface de l'océan sous l'effet des vagues, Comptes Rendus Géosciences, 336, 1121-1130. 
Ardhuin, F., A. D. Jenkins, D. Hauser, A. Reniers, and B. Chapron (2005), Waves and operational oceanography: towards a coherent description of the upper ocean for applications, Eos, 86(4), 37-39.

Barrick, D. E. (1972), First order theory and analysis of MF/HF/VHF scatter from the sea, IEEE Trans. Antennas Propagat., AP-20, 2-10.

Broche, P., J. C. de Maistre, and P. Forget (1983), Mesure par radar décamétrique cohérent des courants superficiels engendrés par le vent, Oceanol. Acta, 6(1), 43-53.

Cafforio, C., P. Guccione, and A. M. Guarnieri (2004a), Doppler centroid estimation for ScanSAR data, IEEE Trans. on Geosci. and Remote Sensing, 42(1), 1-10.

Cafforio, C., P. Guccione, and A. M. Guarnieri (2004b), A spatially selective approach to doppler estimation for frame-based satellite SAR processing, IEEE Trans. on Geosci. and Remote Sensing, 42(6), 1135-1148.

Chapman, R. D., B. L. Gotwols, and R. E. Sterner, II (1994), On the statistics of the phase of microwave backscatter from the ocean surface, J. Geophys. Res., 99(C8), 16,29316,301 .

Chapron, B., F. Collard, and V. Kerbaol (2004), Satellite synthetic aperture radar sea surface doppler measurements, in Proceedings of 2nd workshop on Coastal and Marine Applications of Synthetic Aperture Radar, Svalbard, 8-12 sep, 2003, ESA SP-565, pp. 133-141, ESA Publication Division.

Chelton, D. B., M. G. Schlax, M. H. Freilich, and R. F. Milliff (2004), Satellite measurements reveal persistent small-scale features in ocean winds, Science, 303, 978-983.

Collard, F., F. Ardhuin, and B. Chapron (2005), Extraction of coastal ocean wave fields from SAR images, IEEE J. Oceanic Eng., $X X(\mathrm{XX}), \mathrm{XX}$, in press. 
Csanady, G. T. (1984), The free surface turbulent shear layer, J. Phys. Oceanogr., 14, $402-411$.

Elfouhaily, T., B. Chapron, K. Katsaros, and D. Vandemark (1997), A unified directional spectrum for long and short wind-driven waves, J. Geophys. Res., 102(C7), 15,78115,796 .

Elfouhaily, T., D. R. Thompson, B. Chapron, and D. Vandemark (2001), Improved electromagnetic bias theory: Inclusion of hydrodynamic modulations, J. Geophys. Res., $106(\mathrm{C} 3), 4655-4664$.

Elfouhaily, T. M., and C.-A. Guérin (2004), A critical survey of approximate scattering wave theories from random rough surfaces, Waves in Random Media, 14, 1-40.

Essen, H.-H., and K.-W. Gurgel (2000), On the accuracy of current measurements by means of HF radar, IEEE J. Oceanic Eng., 25(4), 472-480.

Fernandez, D. M., J. F. Vesecky, and C. C. Teague (1998), Measurements of upper ocean surface current shear with high-frequency radar, J. Geophys. Res., 101 (C12), 28,61528,625 .

Fu, L.-L., and B. Holt (1983), Some examples of detection of mesoscale eddies by the SEASAT Synthetic Aperture Radar, J. Geophys. Res., 88(C3), 1844-1852.

Galland, J. C., N. Goutal, and J.-M. Hervouet (1991), TELEMAC: A new numerical model for solving shallow water equations, Adv. Water Resources, 14(3), 138-148.

Goldstein, R. M., and H. A. Zebker (1987), Interferometric radar measurement of ocean surface current, Nature, 328, 707-709.

Graber, H. C., D. R. Thompson, and R. E. Carande (1996), Ocean surface features and currents measured with synthetic aperture radar interferometry and HF radar, J. Geo- 
phys. Res., 101 (C11), 25,813-25,832.

Huang, N. E. (1979), On surface drift currents in the ocean, J. Fluid Mech., 91, 191-208.

Johannessen, J. A., R. A. Shuchman, O. M. Johannessen, K. L. Davidson, and D. R. Lyzenga (1991), Synthetic aperture radar imaging of upper ocean circulation features and wind fronts, J. Geophys. Res., 96(C6), 10,411-10,422.

Johannessen, J. A., R. A. Suchman, G. Digranes, D. R. Lyzenga, C. Wackerman, O. M. Johannessen, and P. W. Vachon (1996), Coastal ocean fronts and eddies imaged with ERS 1, J. Geophys. Res., 101(C3), 6651-6667.

Keller, W. C., W. J. Plant, and G. R. Valenzuela (1986), Observation of breaking ocean waves with coherent microwave radar, in Wave Dynamics and Radio Probing of the Ocean Surface, edited by O. Phillips and K. Hasselmann, pp. 285-293, Plenum Press.

Kudryavtsev, V., D. Hauser, G. Caudal, and B. Chapron (2003a), A semiempirical model of the normalized radar cross-section of the sea surface 1. background model, J. Geophys. Res., 108(C3), 8054, doi:10.1029/2001JCOO1003.

Kudryavtsev, V., D. Hauser, G. Caudal, and B. Chapron (2003b), A semiempirical model of the normalized radar cross-section of the sea surface 2. radar modulation transfer function, J. Geophys. Res., 108(C3), 8055, doi:10.1029/2001JCOO1004.

Kudryavtsev, V. N., V. K. Makin, and B. Chapron (1999), Coupled sea surfaceatmosphere model. 2. spectrum of short wind waves, J. Geophys. Res., 104, 7625-7639.

Lavrenov, I. V. (2003), Wind-waves in oceans: dynamics and numerical simulations, 376 pp., Springer, Berlin.

Longuet-Higgins, M. S. (1957), The statistical analysis of a random, moving surface, Proc. Roy. Soc. Lond. A, 249. 
Longuet-Higgins, M. S. (1987), Lagrangian moments and mass transport in Stokes waves, J. Fluid Mech., 179, 547-555.

Madsen, S. N. (1989), Estimation the doppler centroid of SAR data, IEEE Trans. on Aerospace and Electronic Systems, AES-25(2), 134-140.

Marmorino, G. O., D. R. Thompson, H. C. Graber, and C. L. Trump (1997), Correlation of oceanographic signatures appearing in synthetic aperture radar and interferometric synthetic aperture radar imagery with in situ measurements, J. Geophys. Res., 102(C8), $18,723-18,736$.

Marom, M., R. M. Goldstein, E. B. Thornton, and L. Shemer (1990), Remote sensing of ocean wave spectra by interferometric synthetic aperture radar, Nature, 345, 793-795.

McWilliams, J. C., P. P. Sullivan, and C.-H. Moeng (1997), Langmuir turbulence in the ocean, J. Fluid Mech., 334, 1-30.

Mellor, G., and A. Blumberg (2004), Wave breaking and ocean surface layer thermal response, J. Phys. Oceanogr., 34, 693-698.

Melville, W. K., and R. J. Rapp (1988), The surface velocity in steep and breaking waves, J. Fluid Mech., 169, 1-22.

Moller, D., S. J. Frasier, D. L. Porter, and R. E. McIntosh (1998), Radar-derived interferometric surface currents and their relationship to subsurface current structure, $J$. Geophys. Res., 103(C6), 12,839-12,852.

Munk, W., L. Armi, K. Fischer, and F. Zachariasen (2000), Spirals on the sea, Phil. Trans. Roy. Soc. London A, 456, 1217-1280.

Peirson, W. L., and M. L. Banner (2003), Aqueous surface layer flows induced by microscale breaking wind waves, J. Fluid Mech., 479, 1-38. 
Phillips, O. M. (1985), Spectral and statistical properties of the equilibrium range in wind-generated gravity waves, J. Phys. Oceanogr., 15, 2019-2026.

Plant, W. (2002), A stochastic, multiscale model of microwave backscatter from the ocean, J. Geophys. Res., 107(C9), 3120, doi:10.1029/2001JC000909.

Plant, W. J. (2003), A new interpretation of sea-surface slope probability density functions, J. Geophys. Res., 108(C9), 3295, doi:10.1029/2003JC001870.

Plant, W. J., and W. C. Keller (1990), Evidence of Bragg scattering in microwave Doppler spectra of sea return, J. Geophys. Res., 95(C9), 16,299-16,310.

Portabella, M., A. Stoffelen, and J. A. Johannessen (2002), Toward an optimal inversion method for sythentic aperture radar wind retrieval, J. Geophys. Res., 107(C8), 1-1-113.

Quilfen, Y., B. Chapron, T. Elfouhaily, K. Katsaros, and J. Tournadre (1998), Observation of tropical cyclones by high-resolution scatterometry, J. Geophys. Res., 103, 7767-7786.

Romeiser, R., and D. R. Thompson (2000), Numerical study on the along-track interferometric radar imaging mechanism of oceanic surface currents, IEEE Trans. on Geosci. and Remote Sensing, 38(1), 446-458.

Romeiser, R., H. Breit, M. Eineder, H. Runge, P. Flament, K. de Jong, and J. Vogelzang (2003a), On the suitability of terrasar-x split antenna mode for current measurements by along-track interferometry, in Proceedings of the IGARSS conference, Toulouse, France.

Romeiser, R., H. Breit, M. Eineder, H. Runge, P. Flament, K. de Jong, and J. Vogelzang (2003b), Validation of SRTM-derived surface currents off the Dutch coast by numerical circulation model results, in Proceedings of the IGARSS conference, Toulouse, France. 
Santala, M. J., and E. A. Terray (1992), A technique for making unbiased estimates of current shear from a wave-follower, Deep Sea Res., 39, 607-622.

Shemer, L., M. Marom, and D. Markman (1993), Estimates of currents in the nearshore ocean region using interferometric synthetic aperture radar, J. Geophys. Res., 98(C4), $7001-7010$.

Shuchman, R. (1979), The feasibility of measurement of ocean current detection using sar data, in Proc. of the 13th Int. Symp on Remote Sensing of the Environment, Ann Arbor, edited by R. Beal, pp. 93-103, Applied Physics Laboratory, Johns Hopkins University.

Stewart, R. H., and J. W. Joy (1974), HF radio measurements of surface currents, Deep Sea Res., 21, 1039-1049.

Strong, A. E., and R. J. DeRycke (1973), Ocean current monitoring using a new satellite sensing technique, Science, 182, 482-484.

Terray, E. A., W. M. Drennan, and M. A. Donelan (2000), The vertical structure of shear and dissipation in the ocean surface layer, in Proc. Symp. on Air-Sea Interaction, Sydney, pp. 239-245, University of New South Wales.

Thompson, D. R., B. L. Gotwols, and W. C. Keller (1991), A comparison of Ku-band Doppler measurements at $20^{\circ}$ incidence with predictions from a time-dependent scattering model, J. Geophys. Res., 96(C3), 4947-4955.

Ufermann, S., and R. Romeiser (1999), A new interpretation of multifrequency/multipolarization radar signatures of the Gulf Stream front, J. Geophys. Res., $104(\mathrm{C} 11), 25,697-25,705$.

van der Kooij, M., W. Hughes, and S. Sato (2001), Doppler current velocity measurements: a new dimension to spaceborne data, Unpublished manuscript, available at 
http://www.atlantis-scientific.com/.

van Vledder, G. P., and L. H. Holthuijsen (1993), The directional response of ocean waves to turning winds, J. Phys. Oceanogr., 23, 177-192.

Weber, B. L., and D. E. Barrick (1977), On the nonlinear theory for gravity waves on the ocean's surface. Part I: Derivations, J. Phys. Oceanogr., 7, 3-10.

White, B. S. (1999), Wave action on currents with vorticity, J. Fluid Mech., 386, 329-344.

Winebrenner, D. P., and K. Hasselmann (1988), Specular point scattering contribution to the mean synthetic aperture radar image of the ocean surface, J. Geophys. Res., 93(C8), 9281-9294.

Yu, W., and Z. Zhu (1997), Comparison of Doppler centroid estimation methods in sar, in Proceedings of the IEEE National Aerospace and Electronics Conference (NAECON), 14-17 July 1997, vol. 2, pp. 1015-1018, IEEE. 
Figure 1. Normalised radar cross section $\sigma_{0}$ (grey shades) and Doppler velocity $U_{D}$ (colours), analysed from a wide swath image obtained by ENVISAT on February 6, 2003 at 15:12 UTC. Oceanic fronts appear as sharp gradients of $\sigma_{0}$, while the surface velocity seen by the radar appears to be related to the Gulf Stream.

Figure 2. Dynamic sea surface height for February 5, 2003, corresponding to the time of figure 1, and compiled from all satellite altimeter data over one week. This is the SSALTO-DUACS Near Real Time product, compiled by Collecte Localisation Satellites (CLS). Arrows are visual interpretations of the main features in the dynamic height in terms of surface geostrophic currents.

Figure 3. Same as figure 1, for a different image acquired on March 19, 2004 at 15:12 UTC, covering the Gulf Stream just south of Cape Hatteras.

Figure 4. (a) Surface current predicted by the HYCOM $1 / 8^{\circ}$ model, and (b), surface temperature from the Moderate Resolution Imaging Spectroradiometer (MODIS) on board NASA's Aqua satellite (Daylight Dayly Mean product), for March 19, 2004, within a few hours of the acquisition of the image shown in figure 3 . 
Figure 5. Analysis of Doppler anomalies on a global scale. a, gridded 35 day average (January-February 2004) of Doppler anomaly $f_{\text {Dca }}$, also converted to surface velocity $U_{D}$ (positive for directions away from the satellite) for imagettes acquired by ENVISAT over descending tracks, and thus looking to the West-North-West. The anomaly patterns are well correlated with the general atmospheric circulation of equatorial trade winds and mid-latitude Westerlies, as well as the oceanic equatorial current systems. b, in colors, joint distributions of observed $U_{D}$ and line-of-sight analyzed winds from ECMWF $U_{10 \|}$. Curves show the peak occurrence of $U_{D}$ for a given value of $U_{10 \|}$ (solid black), and models $U_{D, \text { mod }}$ based on equation (B17) with semi-empirical estimates of the tilt and hydrodynamic MTFs, and two estimates of the coefficient $\beta$. An empirical fit to the observation (equation 9) using the C-MOD model [Quilfen et al., 1998] for the NRCS is shown with the red dashed line. All models use upwind values of the NRCS and MTFs for $U_{10 \|}<0$ and downwind values for $U_{10 \|}>0$.

Figure 6. (a) Schematic of the conceptual model of the measurement of surface velocity and the contribution of radar cross-section modulation by waves, and (b) example of parameters over a sinusoidal wave. 
Figure 7. Doppler anomaly in two regions of strong tidal currents (a) off the coast of Normandy, France, on March 92003 (VV polarization image, $31<\theta_{I}<36$ ) and (c), in the Iroise sea, France, November 2002 (HH polarization image $19<\theta_{I}<26$, incidence angles increase with range). Numerical models of near-surface tidal velocities are shown in $\mathrm{b}$ and $\mathrm{d}$.

Figure 8. Significant wave heights and mean directions of westerly swells determined from the March 92003 SAR image (10h24 UTC, figure 7a), and validated with 6 in-situ instruments by Collard et al. [2005]. 


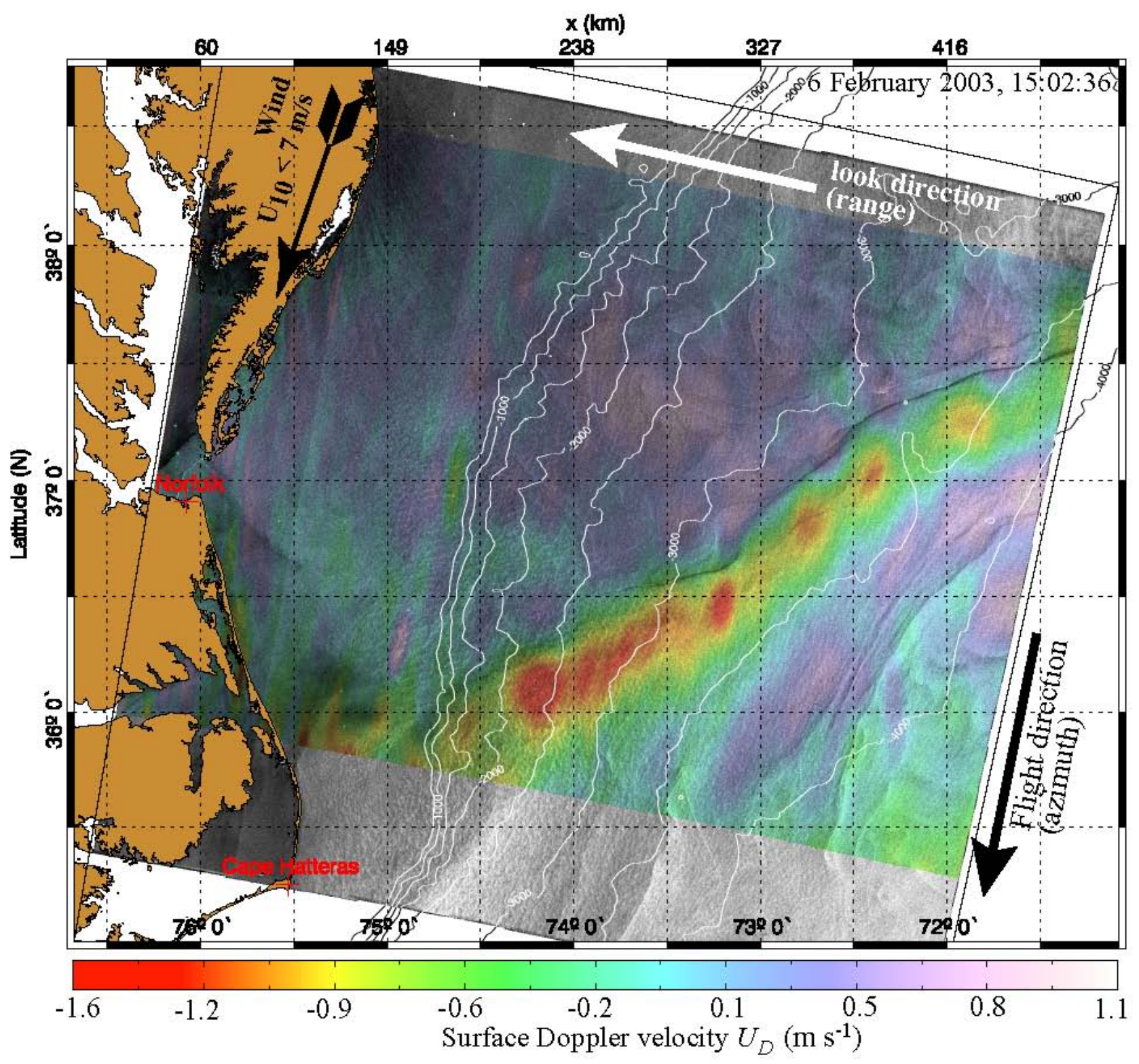




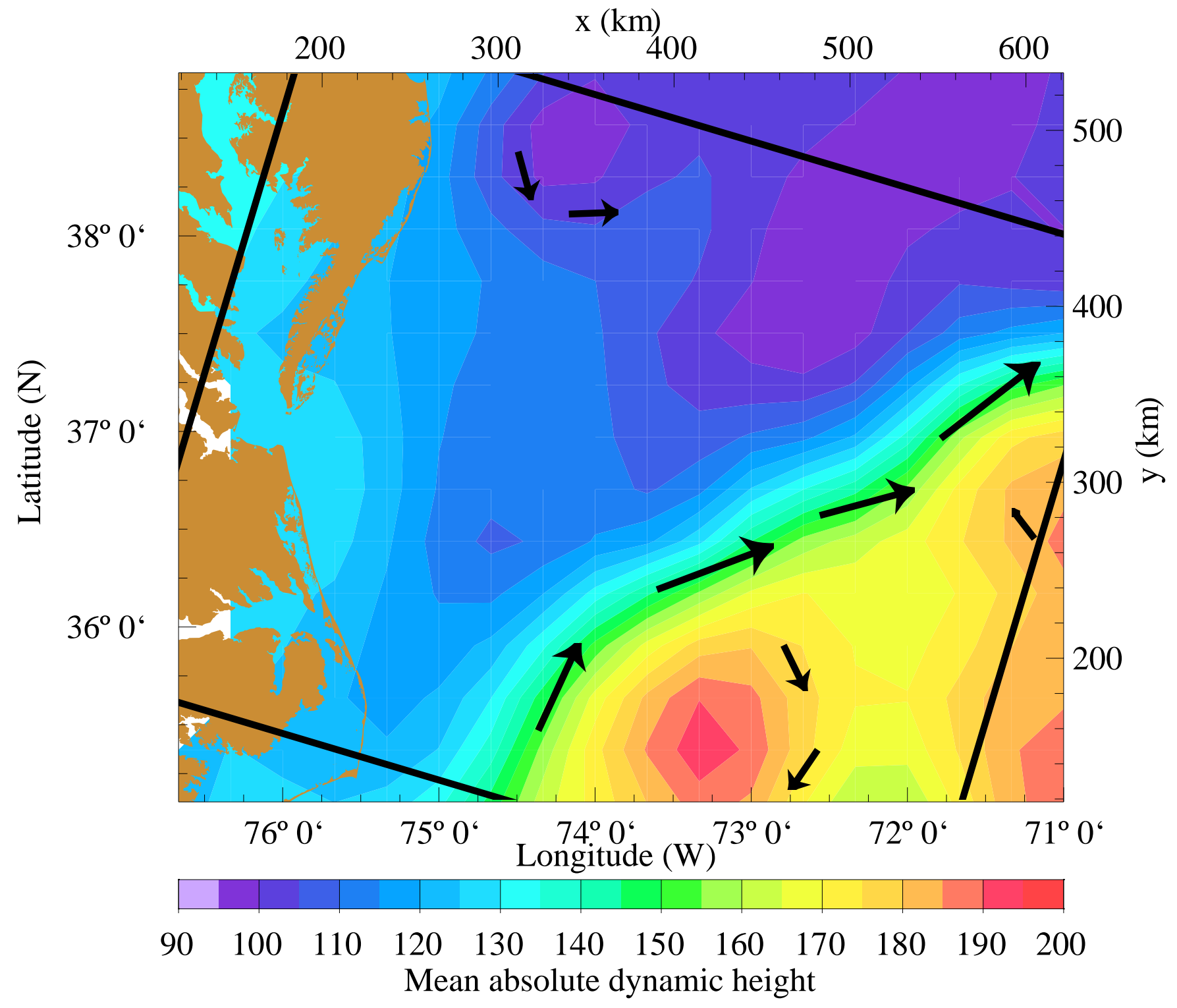




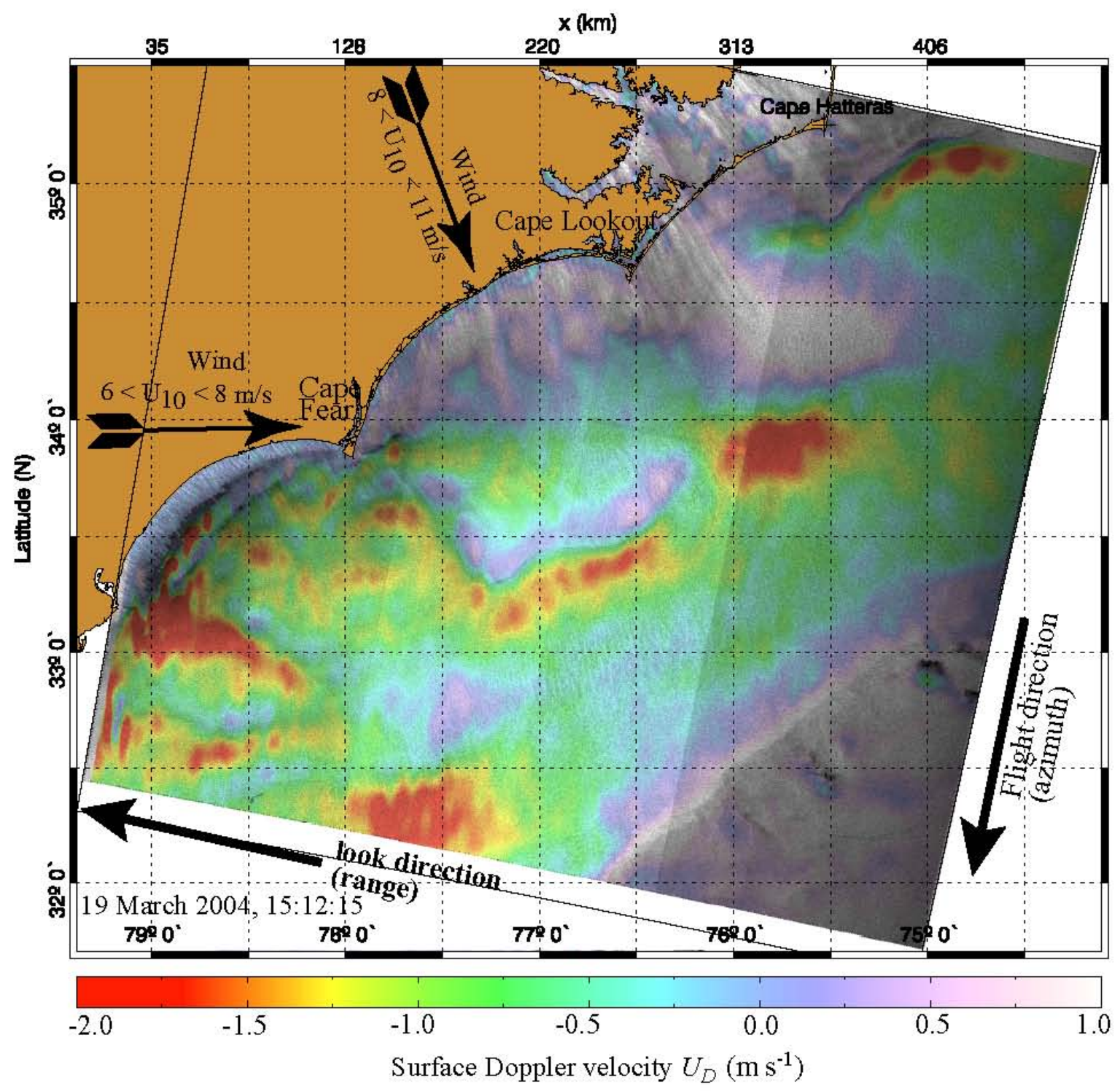



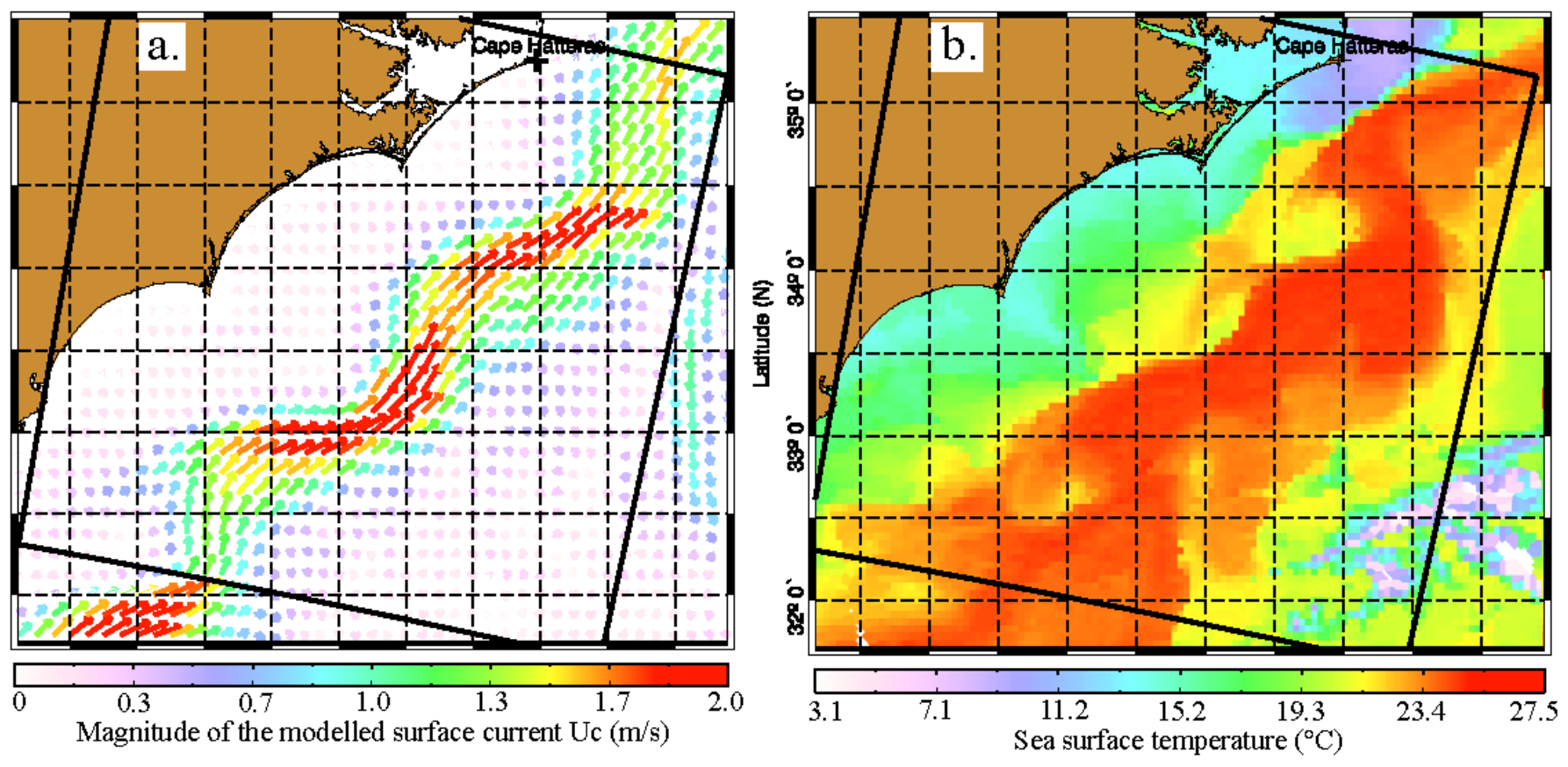


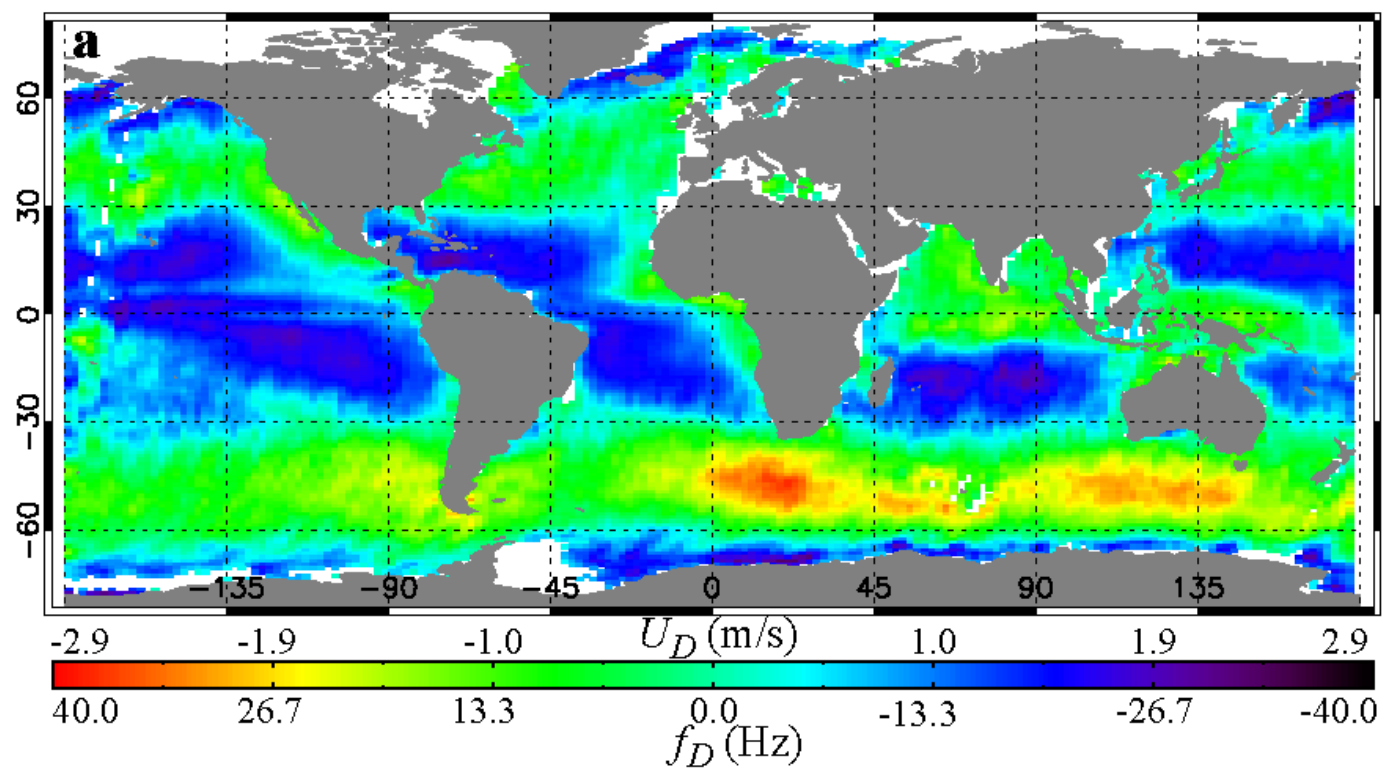

\section{Observations:}

- Peak value of $U_{D}$

\section{Models:}

- $U_{D, \bmod }$ (eq. $\left.1, \beta=0.016\right)$

--- $\quad U_{D, \bmod }$ (eq. A17), $\beta$ from KMC spectrum

\section{Empirical fit:}

- - - fit with CMOD NRCS(eq. 7)

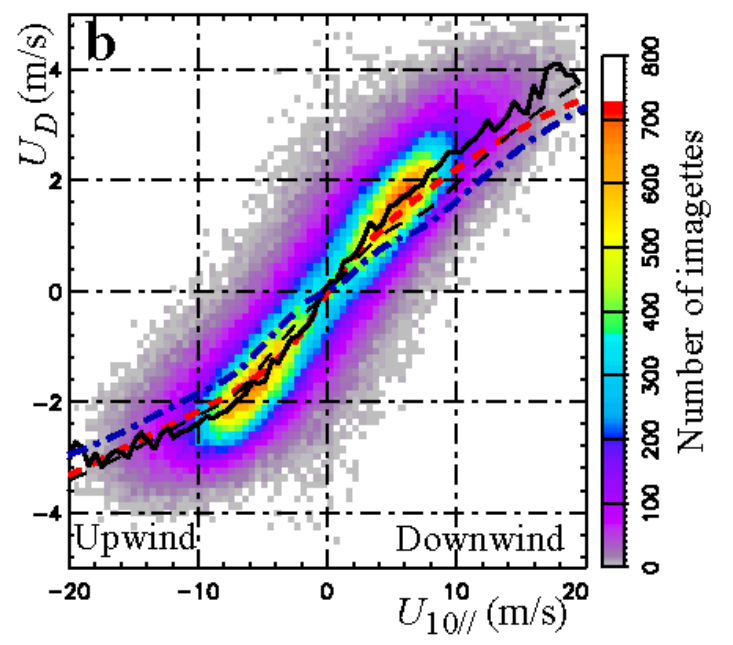




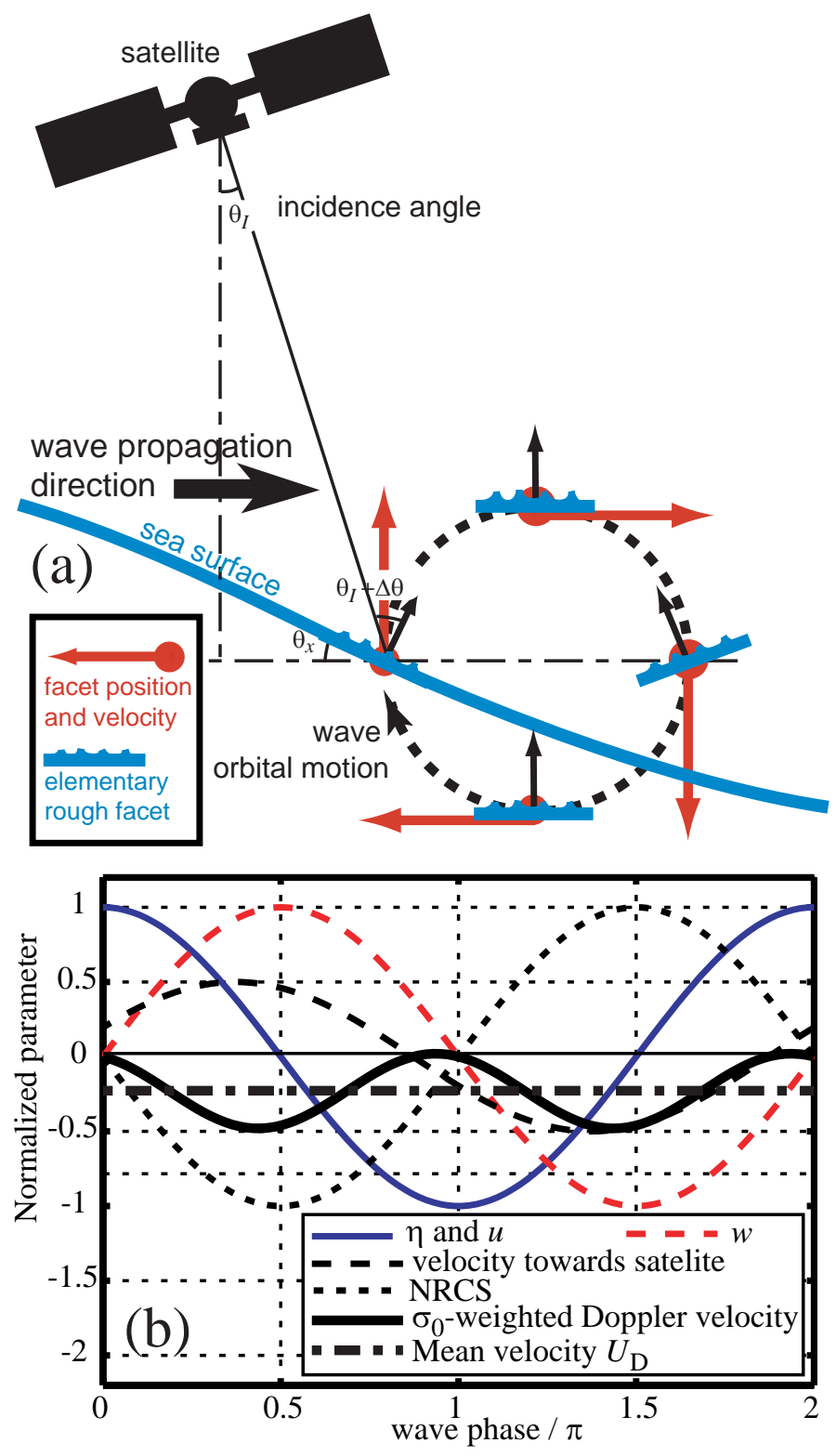



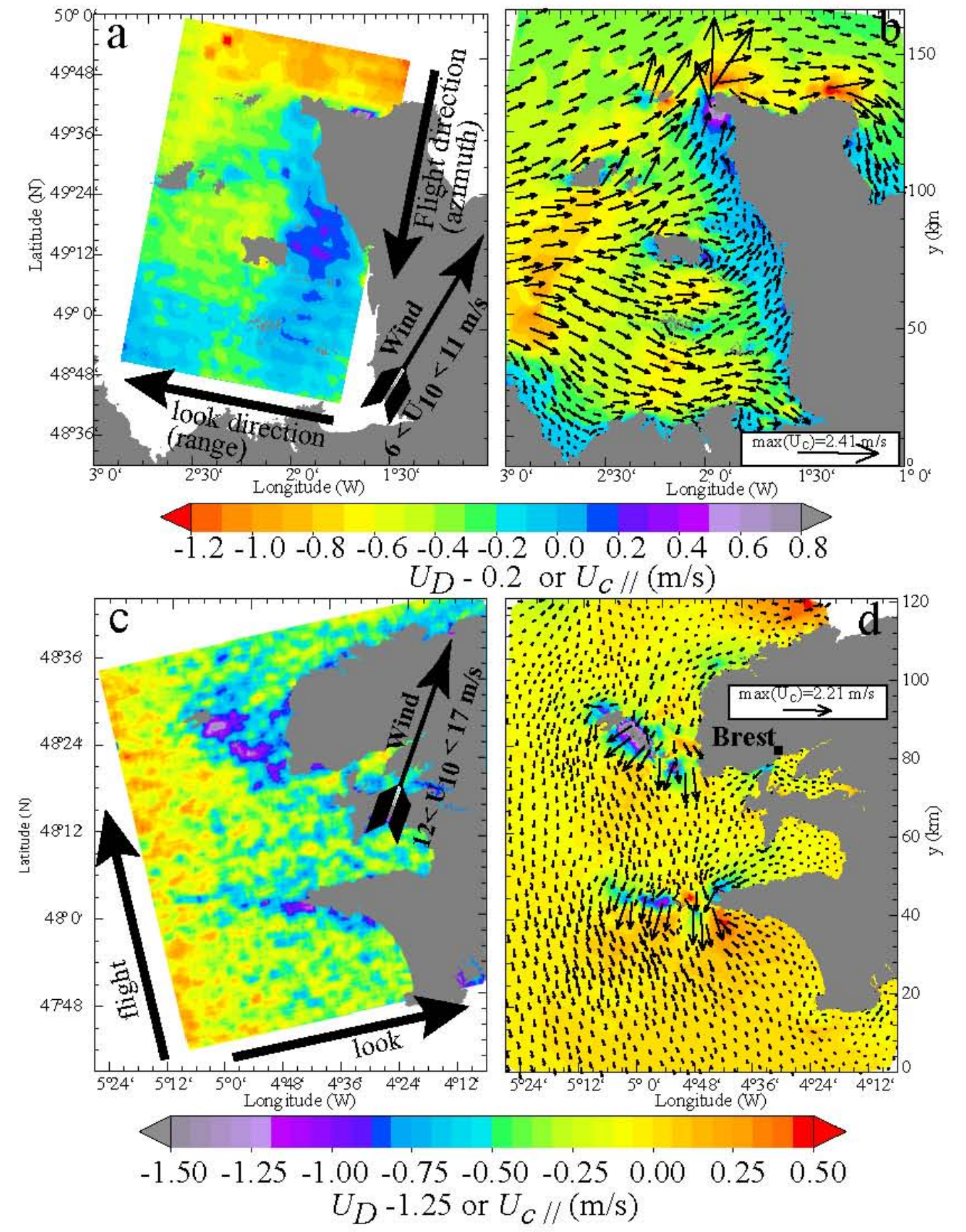


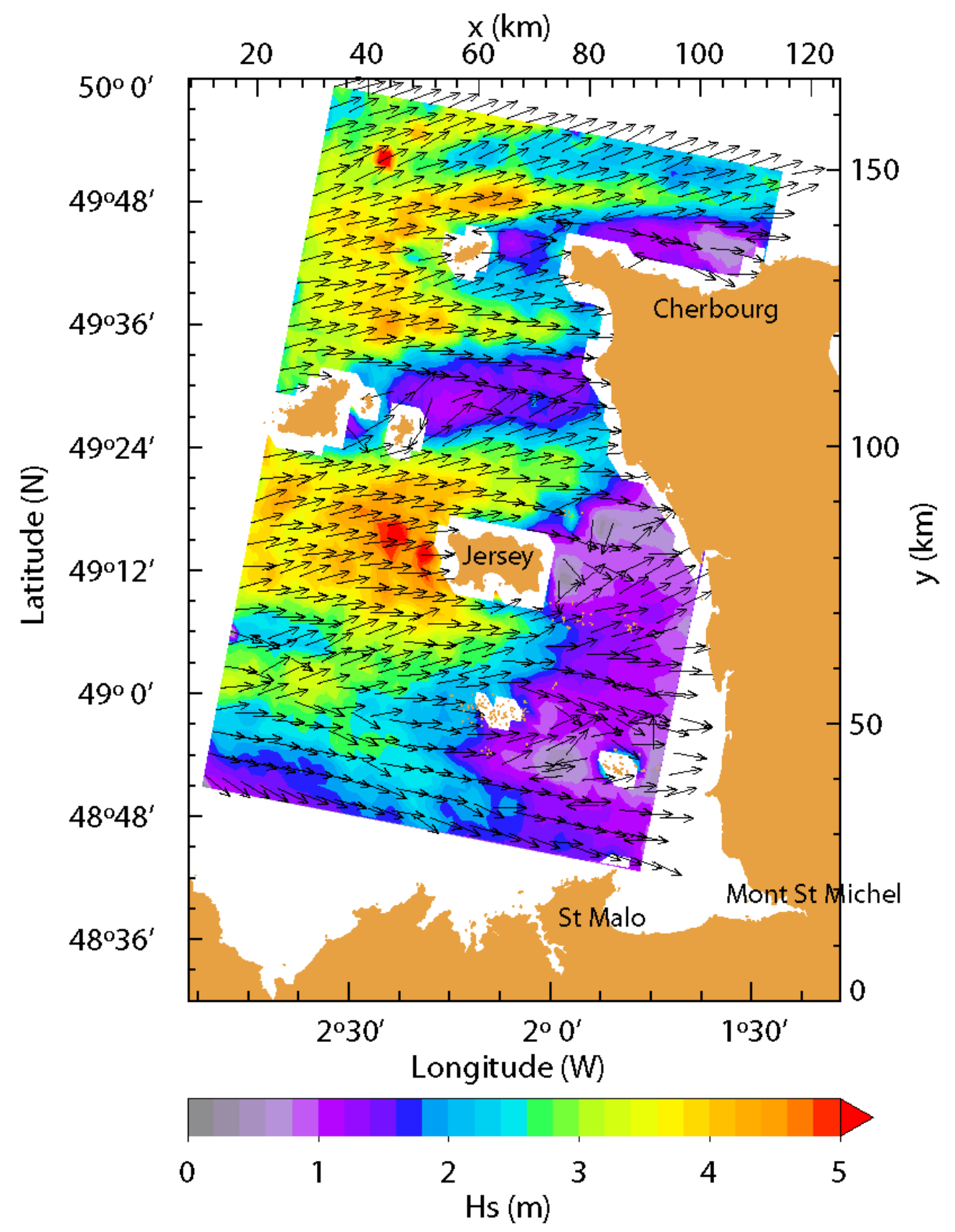

